\title{
Cosine Methods for Second-Order Hyperbolic Equations With Time-Dependent Coefficients*
}

\author{
By Laurence A. Bales, Vassilios A. Dougalis and Steven M. Serbin
}

\begin{abstract}
We analyze efficient, high-order accurate methods for the approximation of the solutions of linear, second-order hyperbolic equations with time-dependent coefficients. The methods are based on Galerkin-type discretizations in space and on a class of fourth-order accurate, two-step, cosine time-stepping schemes. Preconditioned iterative techniques are used to solve linear systems with the same operator at each time step. The schemes are supplemented by single-step high-order starting procedures and need no evaluations of derivatives of operators. $L^{2}$-optimal error estimates are proved throughout.
\end{abstract}

1. Introduction. In this paper we shall study efficient, high-order accurate methods for the approximation of the solutions of linear, second-order hyperbolic equations with time-dependent coefficients. We shall use Galerkin-type discretizations in the space variables and base the time-stepping scheme on a class of fourth-order accurate, two-step methods generated by rational approximations to the cosine; cf., e.g., [3], [4] for the case of time-independent coefficients. The implementation of these "base" schemes requires solving linear systems of equations with operators that vary from time step to time step. Following Douglas, Dupont and Ewing, [9], and Bramble and Sammon, [7], we shall modify the schemes by using preconditioned iterative methods for the approximate solution of the linear systems and thereby only solve linear systems with the same, time-independent operator at every step. If $k$ is the time step, we show that solving $O\left(\ln \left(k^{-1}\right)\right)$ systems at each time step suffices to preserve the overall accuracy and stability of the base schemes.

Preconditioned iterative techniques have been used for second-order hyperbolic problems already by Ewing in [10], where a nonlinear equation is solved by a second-order accurate, two-step time discretization. In addition, one of us, [5], [6], has used such techniques coupled with up to fourth-order accurate single-step discretizations for the problem (1.1) below, written in first-order system form, cf. [2]. These single-step schemes are based on rational approximations to $e^{i x}$ and give rise to quite different time-stepping methods from the ones that we study here. In this paper we take a different approach, discretizing the second-order equation without reducing it first to a first-order system. Our two-step schemes require then, as

Received December 22, 1983; revised June 21, 1984.

1980 Mathematics Subject Classification. Primary 65M15; Secondary 65N30.

* Research supported in part by USARO Grant No. DAAG29-80-K-0056 and by a University of Tennessee faculty research award. 
starting values, not only an approximation of the solution at $t=0$ but also at $t=k$. We supply the latter by using one step of a particular scheme from those analyzed in [5], [6].

We now introduce the problem to be considered. Let $\Omega$ be a bounded domain in $\mathbf{R}^{N}$ with sufficiently smooth boundary $\partial \Omega$ and let $0<t^{*}<\infty$. We shall approximate the (real-valued) solution $u=u(x, t)$, defined on $\bar{\Omega} \times\left[0, t^{*}\right]$, of the initial- and boundary-value problem

$$
\begin{cases}u_{t t}=-L(t) u \equiv \sum_{i, j=1}^{N} \frac{\partial}{\partial x_{i}}\left(a_{i j}(x, t) \frac{\partial u}{\partial x_{j}}\right)-a_{0}(x, t) u \quad \text { in } \Omega \times\left(0, t^{*}\right] \\ u(x, t)=0 & \text { on } \partial \Omega \times\left(0, t^{*}\right] \\ u(x, 0)=u^{0}(x) & \text { in } \Omega \\ u_{t}(x, 0)=u_{t}^{0}(x) & \text { in } \Omega\end{cases}
$$

Here $a_{i j}(x, t)$ and $a_{0}(x, t)$ are sufficiently smooth real-valued functions defined on $\bar{\Omega} \times\left[0, t^{*}\right]$, such that the matrix $\left\{a_{i j}\right\}_{i, j=1}^{N}$ is symmetric and uniformly positive-definite and $a_{0}$ is nonnegative on $\bar{\Omega} \times\left[0, t^{*}\right] . u^{0}(x)$ and $u_{t}^{0}(x)$ are given initial data defined on $\Omega$.

For integer $s \geqslant 0, H^{s}=H^{s}(\Omega)$ will denote the usual Sobolev spaces of real-valued functions on $\Omega$ with norm $\|\cdot\|_{s}$. The inner product on $L^{2}=L^{2}(\Omega)=H^{0}$ is denoted by $(\cdot, \cdot)$ and the associated norm by $\|\cdot\|$. As usual $\stackrel{H}{H}^{1}=\stackrel{\circ}{H}^{1}(\Omega)$ is the subspace of functions in $H^{1}$ that vanish in the sense of trace on $\partial \Omega$.

We shall assume that the operators $L(t)$ defined by $(1.1)$ form, for $t \in\left[0, t^{*}\right]$, a smooth family of unbounded selfadjoint elliptic operators on $L^{2}$ with common domain $D_{L}=H^{2} \cap \stackrel{\circ}{H}^{1}$ and a smooth family of bounded operators from $H^{1+2} \cap D_{L}$ into $H^{\prime}$ for each $l \geqslant 0$. For integer $j \geqslant 0$ we calculate $L^{(j)}(t)=(d / d t)^{j} L(t)$ by differentiating the coefficients of $L$ with respect to $t$. It follows that for $j, l \geqslant 0$, $L^{(j)}(t)$ are also bounded operators from $H^{l+2} \cap D_{L}$ into $H^{l}$.

We shall need the following regularity result for the solution of (1.1). For integer $i \geqslant 0$ let $u^{(i)}=(\partial / \partial t)^{i} u$. Let $u_{0}=u^{0}, u_{1}=u_{t}^{0}$ and, for integer $i \geqslant 2$, define $u_{i}$, the $i$ th time-derivative of $u$ at $t=0$ obtained by differentiating $u_{t t}=-L(t) u$, by

$$
u_{i}=-\sum_{j=0}^{i-2}\left(\begin{array}{c}
i-2 \\
j
\end{array}\right) L^{(i-2-j)}(0) u_{j}
$$

If for $p, m \geqslant 0$, where $p+m \geqslant 2, u_{j} \in D_{L}$ for $0 \leqslant j \leqslant p+m-2$ and $u_{p+m-1} \in$ $\stackrel{\circ}{H}^{1}$, then it is proved, e.g. in [5], that there exists a unique solution of (1.1) such that $u^{(p+m-2)} \in D_{L}, u^{(p)} \in H^{m}$ for $t \in\left[0, t^{*}\right]$ and that there exists a constant $C>0$ such that for $t \in\left[0, t^{*}\right]$ :

$$
\left\|u^{(p)}(t)\right\|_{m} \leqslant C\left(\left\|u^{0}\right\|_{p+m}+\left\|u_{t}^{0}\right\|_{p+m-1}\right) .
$$

In sequel, by assuming smooth and compatible data $u^{0}, u_{t}^{0}$, we shall mean that $u^{0}$ and $u_{t}^{0}$ are such that (1.2) holds for appropriate $p$ and $m$. As is customary, we shall use throughout the paper the symbols $C, C_{i}, C^{\prime}$ to denote generic positive constants, independent of the discretization parameters and the solution $u$ of (1.1). 
We shall let $T(t): L^{2} \rightarrow D_{L}$ denote the solution operator of the elliptic problem $L w=f$ in $\Omega ; w=0$ on $\partial \Omega$. Consequently, we have $L(t)(T(t) f)=f$ for $f \in L^{2}$. For $l \geqslant 0, T(t)$ is then a smooth family of bounded operators from $H^{l}$ into $H^{l+2} \cap D_{L}$. If we put $T^{(j)}(t)=(d / d t)^{j} T(t)$ we have for $t \in\left[0, t^{*}\right]$,

$$
\begin{aligned}
& L^{(1)}(t) T(t)=-L(t) T^{(1)}(t) \text { on } L^{2} \text {, } \\
& T^{(1)}(t) L(t)=-T(t) L^{(1)}(t) \quad \text { on } D_{L} \text {. }
\end{aligned}
$$

By induction it follows that, for each $j \geqslant 0, T^{(j)}(t)$ is a smooth family of bounded operators from $H^{l}$ into $H^{l+2} \cap D_{L}$ for $l \geqslant 0$.

For the space discretization of (1.1) we shall assume, cf. [7], [5], that we have a family of finite-dimensional subspaces $S_{h}$ of $L^{2}$, associated with a parameter $0<h<1$, and a corresponding, sufficiently smooth for $t \in\left[0, t^{*}\right]$, family of bounded operators $T_{h}(t): L^{2} \rightarrow S_{h}$, which are selfadjoint positive-definite on $S_{h}$, positive semidefinite on $L^{2}$, and approximate $T(t)$ in the following way: there exists an integer $r \geqslant 2$, such that, for each $j \geqslant 0$, there exists a constant $C(j)$, independent of $h$, such that for $0 \leqslant l \leqslant r-2$,

$$
\left\|\left(T^{(j)}(t)-T_{h}^{(j)}(t)\right) f\right\| \leqslant C(j) h^{l+2}\|f\|_{l} \quad \text { for } t \in\left[0, t^{*}\right],
$$

if $f \in H^{l}$. Here $T_{h}^{(j)}=(d / d t)^{j} T_{h}$ as usual. We define, on $S_{h}, L_{h}(t)=\left(T_{h}(t)\right)^{-1}$ and put $L_{h}^{(j)}=(d / d t)^{j} L_{h}$. We assume that given $j \geqslant 0$, there exists a constant $C(j)$, independent of $h$, such that

$$
\left|\left(L_{h}^{(j)}(t) \phi, \phi\right)\right| \leqslant C(j)\left(L_{h}(s) \phi, \phi\right) \quad \text { for } \phi \in S_{h}, t, s \in\left[0, t^{*}\right] .
$$

It follows by the symmetry of $L_{h}^{(j)}$ and Lemma 2.1 of [6] that, for $j \geqslant 0$,

$$
\begin{aligned}
& \left|\left(L_{h}^{(j)}(t) \phi, \psi\right)\right| \leqslant C(j)\left(L_{h}(s) \phi, \phi\right)^{1 / 2}\left(L_{h}(s) \psi, \psi\right)^{1 / 2} \\
& \qquad \text { for } \phi, \psi \in S_{h}, t, s \in\left[0, t^{*}\right] .
\end{aligned}
$$

Many well-known Galerkin type methods, including the standard Galerkin method on $S_{h} \subset \stackrel{\circ}{H}^{1}$, satisfy the above conditions. For examples and verification cf., e.g., [8], [11], [5].

We shall also assume that $L_{h}$ satisfies an inverse property, namely that there exists a constant $C_{I}$, independent of $h$, such that

$$
\left(L_{h}(t) \phi, \phi\right) \leqslant C_{I} h^{-2}\|\phi\|^{2} \text { for } \phi \in S_{h}, t \in\left[0, t^{*}\right] .
$$

(1.7) has a number of useful consequences. One may show, cf., e.g., [11], [5], that it implies that for $j \geqslant 0$ there exist constants $C(j)$ such that for $s, t \in\left[0, t^{*}\right]$,

$$
\left\|L_{h}^{(j)}(t) T_{h}(s)\right\|, \quad\left\|T_{h}(s) L_{h}^{(j)}(t) P\right\| \leqslant C(j),
$$

where $P: L^{2} \rightarrow S_{h}$ is the orthogonal $L_{2}$-projection operator onto $S_{h}$. In addition, it follows from (1.7) that for $j \geqslant 0$,

$$
\left\|L_{h}^{(j)}(t) P\right\| \leqslant C(j) h^{-2}, \quad t \in\left[0, t^{*}\right],
$$

and

$$
\left\|L_{h}^{(j)}(t) \phi\right\| \leqslant C(j)\left\|L_{h}(s) \phi\right\| \quad \text { for } \phi \in S_{h}, s, t \in\left[0, t^{*}\right] .
$$


To approximate the solution of (1.1) one may define as an intermediate stage the semidiscrete approximation $u_{h}:\left[0, t^{*}\right] \rightarrow S_{h}$ that satisfies (with $D_{t}=d / d t$ )

$$
D_{t}^{2} u_{h}(t)+L_{h}(t) u_{h}(t)=0, \quad t \in\left(0, t^{*}\right] .
$$

If (1.11) is supplemented with suitable initial data $u_{h}(0), D_{t} u_{h}(0)$, it may be shown, cf. [5], that $\left\|u_{h}(t)-u(t)\right\|=O\left(h^{r}\right)$ for $t \in\left[0, t^{*}\right]$. We shall not use the semidiscrete approximation at all in this work, except in order to motivate the time-stepping schemes.

Our full discretization of (1.1) will be based on real-valued rational approximations $r(x)$ to $\cos x$ for $x \in \mathbf{R}^{1}$, cf. [12], [3], [4]. The rational functions that we shall consider are of the form

$$
\begin{aligned}
& r(x)=\frac{1+p_{1} x^{2}+p_{2} x^{4}}{1+q_{1} x^{2}+q_{2} x^{4}}, \\
& \text { with } 1+q_{1} x^{2}+q_{2} x^{4}>0 \text { for } x \in \mathbf{R}^{1} \text { and } q_{2} \neq 0 .
\end{aligned}
$$

We assume that $r(x)$ is a fourth-order accurate approximation to $\cos x$, i.e., that it satisfies, for $|x|$ sufficiently small,

$$
|r(x)-\cos x| \leqslant C x^{6}
$$

We shall also assume that the "stability condition"

$$
|r(x)| \leqslant 1 \quad \text { for } x \in \mathbf{R}^{1},
$$

is satisfied.

As a parenthetical remark we should note here that the convergence analysis of Sections 2-5 can be applied (with obvious modifications, although we shall not state a formal result) to the much easier case of rational approximations of the form $r(x)=\left(1+p_{1} x^{2}\right) /\left(1+q_{1} x^{2}\right)$. These lead, in general, to second-order accurate schemes with the exception of the case $q_{1}=1 / 12, p_{1}=-5 / 12$, corresponding to the Størmer-Numerov method, which is fourth-order accurate but satisfies (1.14) only for $|x|$ sufficiently small. Our analysis can also be easily extended to cover, under stability restrictions on the discretization parameters of the form " $k h^{-1}$ small", "conditionally stable" methods, i.e., cases for which (1.14) holds for $|x| \leqslant \alpha<\infty$. However, it seems that our analysis cannot be extended, without restrictions of the form " $k h^{-\alpha}$ small", $\alpha \geqslant 1$, to methods that result from rational functions $r(x)$ with even polynomials of degree higher than four as their numerator or denominator. Similarly, we do not treat the case of special methods of the form (1.12) of order greater than fourth (there exists a one-parameter family of sixth order and one eighth-order method that at most satisfy (1.14) for small $|x|$-if they do so at all-), since such methods would require appropriate higher-order single-step starting procedures for which no theory is available without similar restrictions on $k$ and $h$.

We turn now to a more detailed description of the rational approximations that satisfy (1.12)-(1.14). It is not hard to see that fourth-order accuracy, i.e., condition (1.13), implies that the coefficients $p_{1}, p_{2}$ must be related to $q_{1}, q_{2}$ by the equations

$$
\begin{aligned}
& p_{1}=q_{1}-1 / 2, \\
& p_{2}=q_{2}-q_{1} / 2+1 / 24
\end{aligned}
$$




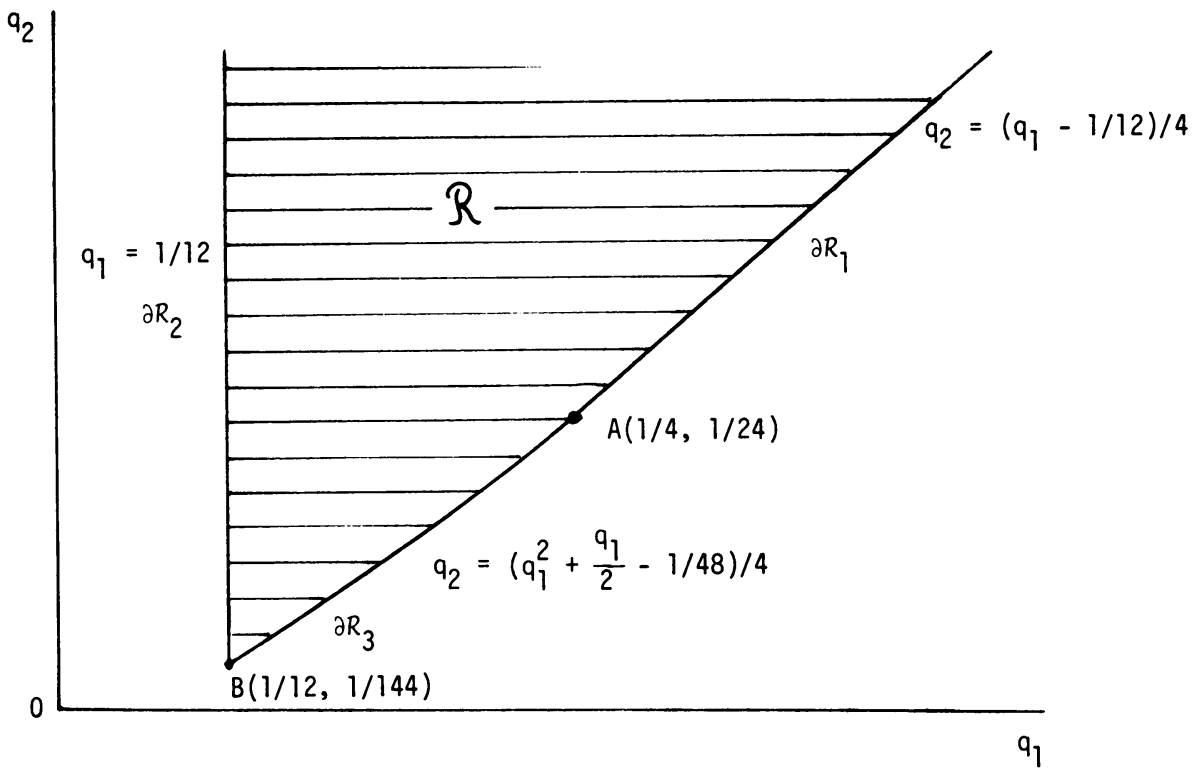

FIGURE 1

Stability region for $q_{1}, q_{2}$

A straightforward elementary analysis shows now that if $p_{1}, p_{2}$ are given by (1.15), (1.16), then (1.14) is equivalent to the condition

$$
\left(q_{1}, q_{2}\right) \in \overline{\mathscr{R}},
$$

where the region $\mathscr{R}$, a subset of the $q_{1}, q_{2}>0$ quarter plane is shown in Figure 1 . We put

$$
\overline{\mathscr{R}}=\mathscr{R} \cup \partial \mathscr{R} \quad \text { and } \quad \partial \mathscr{R}=\partial \mathscr{R}_{1} \cup \partial \mathscr{R}_{2} \cup \partial \mathscr{R}_{3} \cup\{A\} \cup\{B\} \text {, }
$$

where $A=(1 / 4,1 / 24), \quad B=(1 / 12,1 / 144), \quad \partial \mathscr{R}_{1}=\left\{\left(q_{1}, q_{2}\right): q_{1}>1 / 4, q_{2}=\right.$ $\left.\left(q_{1}-1 / 12\right) / 4\right\}, \quad \partial \mathscr{R}_{2}=\left\{\left(q_{1}, q_{2}\right): q_{1}=1 / 12, \quad q_{2}>1 / 144\right\}, \quad \partial \mathscr{R}_{3}=\left\{\left(q_{1}, q_{2}\right):\right.$ $\left.q_{2}=\left(q_{1}^{2}+q_{1} / 2-1 / 48\right) / 4,1 / 12<q_{1}<1 / 4\right\}$. In $\overline{\mathscr{R}}$ we have $|r(x)| \leqslant 1$ for $x \in$ $\mathbf{R}^{1}$. In particular, in $\mathscr{R}$ we always have $-1+\delta<r(x)<1$ for some $\delta>0$ for all $x \in \mathbf{R}^{1}$ and also $\lim _{|x| \rightarrow \infty}|r(x)|<1$, while $\lim _{x \rightarrow \infty} r(x)=-1$ on $\partial \mathscr{R}_{1}$, $\lim _{x \rightarrow \infty} r(x)=1$ on $\partial \mathscr{R}_{2}$, whereas on $\partial \mathscr{R}_{3}, r\left(x_{0}\right)=-1$ at $x_{0}= \pm\left(8 /\left(1-4 q_{1}\right)\right)^{1 / 2}$. For further reference we also note that if (1.15) and (1.16) are satisfied, then the following hold:

$$
\left\{\begin{array}{l}
p_{2}+q_{2}>0, \quad q_{2}-p_{2}>0 \text { in } \mathscr{R}, \\
q_{1}-p_{1}>0 \text { in } \mathscr{R}, \quad q_{2}-p_{2}=0 \text { on } \partial \mathscr{R}_{2} \cup\{B\}, \\
p_{2}+q_{2}=0 \text { on } \partial \mathscr{R}_{1} \cup\{A\}, \\
q_{2}+p_{2}=\left(q_{1}+p_{1}\right)^{2} / 8 \text { on } \partial \mathscr{R}_{3} \cup\{A\} \cup\{B\} .
\end{array}\right.
$$

Many useful schemes are associated with rational approximation $r(x)$ that satisfy (1.15)-(1.17). For example, the point $B$ represents the approximation to the cosine obtained as the real part of the $(2,2)$ Padé rational approximation to $e^{i x}$. The special approximations $r(x)$ with denominators $\left(1+\beta x^{2}\right)^{2}, \beta>0$, considered in [3], [4], that are especially effective for problems with time-independent coefficients, correspond to $q_{1}=2 \beta, q_{2}=\beta^{2}$ (with $p_{1}, p_{2}$ given by (1.15), (1.16)), i.e., lying on the parabola $q_{2}=q_{1}^{2} / 4$ which crosses $\partial \mathscr{R}_{1}$ and lies inside $\overline{\mathscr{R}}$ for $q_{1}=2 \beta \geqslant 1 / 2+1 / \sqrt{6}$. 
To discretize now (1.11) in time, we assume that $u_{h}(t)$ is sufficiently smooth, let $k>0$ be the time step and put $t^{n}=n k, n=0,1,2, \ldots, J$ with $t^{J}=t^{*}$ for simplicity. The fully discrete methods generated by the rational approximation $r(x)$ to $\cos x$, henceforth referred to as "cosine methods" may be derived as follows. If we approximate $\cosh z \equiv \cos (i z)$ by $r(i z)$ in the formal expansion $y\left(t^{n+1}\right)+y\left(t^{n-1}\right)=$ $2 \cosh \left(k D_{t}\right) y\left(t^{n}\right)$ and replace $y(t)$ by $u_{h}(t)$, we obtain, from (1.12) and (1.13), for $1 \leqslant n \leqslant J-1$,

$$
\begin{aligned}
& \left(I-q_{1} k^{2} D_{t}^{2}+q_{2} k^{4} D_{t}^{4}\right)\left(u_{h}^{n+1}+u_{h}^{n-1}\right) \\
& \quad=2\left(I-p_{1} k^{2} D_{t}^{2}+p_{2} k^{4} D_{t}^{4}\right) u_{h}^{n}+O\left(k^{6} u_{h}^{(6)}\right) .
\end{aligned}
$$

In (1.19) and in sequel, we let $y^{n}=y\left(t^{n}\right), 0 \leqslant n \leqslant J$, and $y^{(i)}=D_{t}^{i} y$ for a smooth function $y(t)$ defined on $\left[0, t^{*}\right]$. Now, differentiating (1.11) yields

$$
u_{h}^{(4)}(t)=\left(L_{h}^{2}(t)-L_{h}^{(2)}(t)\right) u_{h}(t)-2 L_{h}^{(1)}(t) u_{h}^{(1)}(t)
$$

Introducing the polynomials $q(\tau)=1+q_{1} \tau+q_{2} \tau^{2}, p(\tau)=1+p_{1} \tau+p_{2} \tau^{2}$ and the notation $L_{h}\left(t^{n}\right)=L_{h}^{n}, L_{h}^{(j)}\left(t^{n}\right)=L_{h}^{(j) n}$ for $j \geqslant 0, Q^{n}=q\left(k^{2} L_{h}^{n}\right), P^{n}=p\left(k^{2} L_{h}^{n}\right)$ for $0 \leqslant n \leqslant J$, we obtain, by (1.19) and (1.20), for $1 \leqslant n \leqslant J-1$,

$$
\begin{aligned}
& Q^{n+1} u_{h}^{n+1}+Q^{n-1} u_{h}^{n-1}-2 P^{n} u_{h}^{n} \\
&= k^{4} q_{2}\left(L_{h}^{(2) n+1} u_{h}^{n+1}+L_{h}^{(2) n-1} u_{h}^{n-1}-2 L_{h}^{(2) n} u_{h}^{n}\right) \\
&+2 k^{4} q_{2}\left(L_{h}^{(1) n+1} u_{h}^{(1) n+1}+L_{h}^{(1) n-1} u_{h}^{(1) n-1}-2 L_{h}^{(1) n} u_{h}^{(1) n}\right) \\
&+k^{4}\left(q_{1}-\frac{1}{12}\right)\left(L_{h}^{(2) n} u_{h}^{n}+2 L_{h}^{(1) n} u_{h}^{(1) n}\right)+O\left(k^{6} u_{h}^{(6)}\right) .
\end{aligned}
$$

Since we are interested in fourth-order accurate methods, i.e., of $O\left(k^{6}\right)$ local accuracy, we see that the first two terms in the right-hand side of (1.21), being of the form $C k^{4}\left(v^{n+1}-2 v^{n}+v^{n-1}\right), v$ smooth, may be dropped without affecting accuracy. We also want to compute with a scheme that involves only approximations to the values of $u_{h}^{n}$ and not of its derivative $u_{h}^{(1) n}$. We shall therefore replace, in the last term of the right-hand side of (1.21), $u_{h}^{(1) n}$ by its $O\left(k^{2}\right)$ backward-difference approximation:

$$
\frac{1}{k}\left(u_{h}^{n}-u_{h}^{n-1}\right)+\frac{k}{2} u_{h}^{(2) n}=\frac{1}{k}\left(u_{h}^{n}-u_{h}^{n-1}\right)-\frac{k}{2} L_{h}^{n} u_{h}^{n}
$$

where we have used (1.11). Thus we arrive at the following fully discrete scheme for the approximation of $u^{n}, 0 \leqslant n \leqslant J$, where $u$ is the solution of (1.1). For $U^{0}, U^{1}$ given elements of $S_{h}$, that will be constructed in sequel as suitable approximations to $u(0)$ and $u(k)$, we seek $\left\{U^{n}\right\}_{n=2}^{J}$ in $S_{h}$ as solutions of the equations

$$
\begin{aligned}
& Q^{n+1} U^{n+1}+Q^{n-1} U^{n-1}-2 P^{n} U^{n} \\
& =k^{4}\left(q_{1}-\frac{1}{12}\right)\left\{L_{h}^{(2) n} U^{n}+2 L_{h}^{(1) n}\left[\frac{1}{k}\left(U^{n}-U^{n-1}\right)-\frac{k}{2} L_{h}^{n} U^{n}\right]\right\}, \\
& 1 \leqslant n \leqslant J-1 .
\end{aligned}
$$

Due to the positivity of $q(\tau)$ for $\tau \geqslant 0$, the selfadjoint operators $Q^{n}$ are positivedefinite on $S_{h}$ for $0 \leqslant n \leqslant J$. Hence (1.22) possesses for each $n, 1 \leqslant n \leqslant J-1$, a 
unique solution $U^{n+1}$ in $S_{h}$. The scheme (1.22) will be referred to in sequel as the base scheme. In Section 2 we shall study its consistency and stability. It will follow from Theorem 2.1 that, if (1.15)-(1.17) hold, then, under the conditions that $k h^{-1}$ be bounded as $k, h \rightarrow 0$, if $\left(q_{1}, q_{2}\right) \in \partial \mathscr{R}_{1} \cup \partial \mathscr{R}_{2} \cup\{A\}, k h^{-1}$ be appropriately small if $\left(q_{1}, q_{2}\right) \in \partial \mathscr{R}_{3} \cup\{B\}$ and unconditionally otherwise and if $u^{0}, u_{t}^{0}$ are sufficiently smooth and compatible,

$$
\max _{0 \leqslant n \leqslant J}\left\|u^{n}-U^{n}\right\|=O\left(k^{4}+h^{r}\right)+\varepsilon^{0,1}
$$

holds. Here $\varepsilon^{0,1}$ is an error term that depends on the choice of the initial data $U^{0}, U^{1}$. This choice is discussed in Section 3 where it is shown that if $U^{0}$ is defined as the "elliptic projection" of $u^{0}$ and $U^{1}$ is constructed by means of a single-step method from those analyzed in [5], [6], then, for $u^{0}, u_{t}^{0}$ sufficiently smooth and compatible, the optimal estimate $\varepsilon^{0,1}=O\left(k^{4}+h^{r}\right)$ holds as well.

Solving for $U^{n+1}$ by (1.22) necessitates inverting, for each $n$, the operator $Q^{n+1}$ that varies with $n$. Using preconditioned iterative techniques, following [9], [7], we show in Section 4 how to modify the base scheme so that the resulting method needs solving $O\left(\ln \left(k^{-1}\right)\right)$ systems with the same operator for every $n$, and preserves the optimal error estimate. The starting scheme used to compute $U^{1}$ may be also similarly modified so that the overall method will be quite efficient.

In (1.22) we note that in general (i.e., for cosine methods with $q_{1} \neq 1 / 12$ ), we need to calculate, for each $n$, the time-derivatives $L_{h}^{(i) n}, i=1,2$, of the operator $L_{h}$. To avoid such calculations, we replace in Section 5 these derivatives by centered-difference quotients that involve only values of $L_{h}^{n}$ for $0 \leqslant n \leqslant J$. (The same thing is done in the starting scheme that defines $U^{1}$; however, the computation of $L_{h}^{(1) 0}$ is needed.) We prove optimal-error estimates for the resulting method that is then coupled with preconditioned iterative techniques for solving the attendant linear systems at each time step.

2. Consistency and Stability of the Base Scheme. For easy reference we rewrite here the base scheme (1.22) as follows. Given $U^{0}, U^{1}$ in $S_{h}$, we seek $\left\{U^{n}\right\}_{n=2}^{J}$ in $S_{h}$ satisfying, for $1 \leqslant n \leqslant J-1$,

$$
\begin{aligned}
\mathscr{S}^{n}\left(U^{n}\right) \equiv & Q^{n+1} U^{n+1}+Q^{n-1} U^{n-1}-2 P^{n} U^{n} \\
& -k^{4}\left(q_{1}-\frac{1}{12}\right)\left[L_{h}^{(2) n} U^{n}+2 L_{h}^{(1) n}\left(\frac{1}{k}\left(U^{n}-U^{n-1}\right)-\frac{k}{2} L_{h}^{n} U^{n}\right)\right]=0 .
\end{aligned}
$$

We shall compare $U^{n}$ with the "elliptic projection" $W^{n}$ of $u^{n}$, which is defined as follows. Let $P_{I}(t)=T_{h}(t) L(t): D_{L} \rightarrow S_{h}$ for $t \in\left[0, t^{*}\right]$. As is well-known, it follows from our assumptions that if $v \in H^{l+2} \cap D_{L}$ for some $0 \leqslant l \leqslant r-2$, then

$$
\|v-P v\| \leqslant\left\|v-P_{I} v\right\| \leqslant C h^{1+2}\|v\|_{l+2} \text { for } t \in\left[0, t^{*}\right] .
$$

Let $W(t)=P_{I}(t) u(t)$. Then, it is proved, e.g., in [7], [6], that if $m \geqslant 0,0 \leqslant l \leqslant r-2$ and $u^{0}, u_{t}^{0}$ are sufficiently smooth and compatible, then

$$
\left\|u^{(m)}(t)-W^{(m)}(t)\right\| \leqslant C(m) h^{l+2}\left(\left\|u^{0}\right\|_{m+l+2}+\left\|u_{t}^{0}\right\|_{m+l+1}\right)
$$

$$
\text { for } t \in\left[0, t^{*}\right] \text {, }
$$


and that it follows from (1.8) that if $m \geqslant 0$

$$
\left\|L_{h}(s) W^{(m)}(t)\right\| \leqslant C\left(\left\|u^{0}\right\|_{m+2}+\left\|u_{t}^{0}\right\|_{m+1}\right) \text { for } t, s \in\left[0, t^{*}\right] .
$$

In view of (2.3) we shall be interested in comparing $U^{n}$ with $W^{n}$. Let $E^{n}=U^{n}-$ $W^{n}$. Then (2.1) yields the following error equation for the base scheme

$$
\mathscr{S}^{n}\left(E^{n}\right)=-\mathscr{S}^{n}\left(W^{n}\right), \quad 1 \leqslant n \leqslant J-1 .
$$

To estimate $E^{n}$ we first investigate the consistency of the base scheme. To this effect we state the following lemma, whose proof follows easily from Taylor's theorem and (1.13) (i.e., (1.15) and (1.16)) and (1.2).

Lemma 2.1. Let $u^{0}, u_{t}^{0}$ be compatible with $u^{0} \in H^{6}, u_{t}^{0} \in H^{5}$. Then for some constant $C$, independent of $h, k$, and $u$, and for $1 \leqslant n \leqslant J-1$,

$$
\begin{aligned}
& \left\|q\left(-k^{2} D_{t}^{2}\right)\left(u^{n+1}+u^{n-1}\right)-2 p\left(-k^{2} D_{t}^{2}\right) u^{n}\right\| \\
& \leqslant C k^{6} \sup _{t^{n-1} \leqslant \tau \leqslant t^{n+1}}\left\|u^{(6)}(\tau)\right\| \leqslant C k^{6}\left(\left\|u^{0}\right\|_{6}+\left\|u_{t}^{0}\right\|_{5}\right) .
\end{aligned}
$$

For the proof of the main consistency result we shall need the following technical fact.

Lemma 2.2. Let $v \in H^{l}$ for some $0 \leqslant l \leqslant r-2$. Then, for $j=1,2$ there exist constants $C(j)$, independent of $h$ and $v$, such that

$$
\begin{aligned}
& \left|\left(\left[L_{h}^{(j)}(t) T_{h}(t)-L^{(j)}(t) T(t)\right] v, \phi\right)\right| \\
& \quad \leqslant C(j) h^{l+2}\|v\|\|\|_{h}(t) \phi \| \quad \text { for } \phi \in S_{h}, t \in\left[0, t^{*}\right] .
\end{aligned}
$$

Proof. Suppressing the dependence on $t$ we have for $j=1$ and $\phi \in S_{h}$, using (1.3), its $T_{h}, L_{h}$-analog, (1.4) and (2.2), that

$$
\begin{aligned}
& \left|\left(\left(L_{h}^{(1)} T_{h}-L^{(1)} T\right) v, \phi\right)\right|=\left|\left(\left(T_{h}^{(1)}-T_{h} L T^{(1)}\right) v, L_{h} \phi\right)\right| \\
& \leqslant\left(\left\|\left(T_{h}^{(1)}-T^{(1)}\right) v\right\|+\left\|\left(I-P_{I}\right) T^{(1)} v\right\|\right)\left\|L_{h} \phi\right\| \leqslant C h^{l+2}\|v\|,\left\|L_{h} \phi\right\| .
\end{aligned}
$$

For $j=2$ we note that differentiating (1.3) and its $T_{h}, L_{h}$-analog yields on $H^{l}$

$$
\begin{aligned}
L_{h}^{(2)} T_{h} & -L^{(2)} T=2\left(L_{h}^{(1)} T_{h} L_{h}^{(1)} T_{h}-L^{(1)} T L^{(1)} T\right)-\left(L_{h} T_{h}^{(2)}-L T^{(2)}\right) \\
& =2 L_{h}^{(1)} T_{h}\left(L_{h}^{(1)} T_{h}-L^{(1)} T\right)+2\left(L_{h}^{(1)} T_{h}-L^{(1)} T\right) L^{(1)} T-\left(L_{h} T_{h}^{(2)}-L T^{(2)}\right) .
\end{aligned}
$$

It follows from (2.7) for $j=1,(1.10),(1.4),(2.2)$ for $v \in H^{l}, \phi \in S_{h}$, that

$$
\begin{aligned}
& \left|\left(\left(L_{h}^{(2)} T_{h}-L^{(2)} T\right) v, \phi\right)\right| \\
& \quad=\mid 2\left(\left(L_{h}^{(1)} T_{h}-L^{(1)} T\right) v, T_{h} L_{h}^{(1)} \phi\right)+2\left(\left(L_{h}^{(1)} T_{h}-L^{(1)} T\right) L^{(1)} T v, \phi\right) \\
& \quad-\left(\left(T_{h}^{(2)}-T^{(2)}\right) v, L_{h} \phi\right)-\left(\left(I-P_{I}\right) T^{(2)} v, L_{h} \phi\right) \mid \\
& \quad \leqslant C h^{l+2}\|v\|\left\|_{l}\right\| L_{h} \phi \| . \quad \square
\end{aligned}
$$

We now come to our main consistency result.

Proposition 2.1. Let $u^{0}, u_{t}^{0}$ be compatible with $u^{0} \in H^{\mu}$ and $u_{t}^{0} \in H^{\mu-1}, \mu=$ $\max (6, r+2)$. Then, there exists a constant $C$, independent of $h, k$ and $u$, such that for $\phi \in S_{h}, 1 \leqslant n \leqslant J-1$,

$$
\begin{aligned}
\left|\left(\mathscr{S}^{n}\left(W^{n}\right), \phi\right)\right| \leqslant & C k^{2}\left\{k^{4}\left(\left\|u^{0}\right\|_{6}+\left\|u_{t}^{0}\right\|_{5}\right)+h^{r}\left(\left\|u^{0}\right\|_{r+2}+\left\|u_{t}^{0}\right\|_{r+1}\right)\right\} \\
& \times\left\{\|\phi\|+k^{2}\left\|L_{h}^{n} \phi\right\|\right\} .
\end{aligned}
$$


Proof. From (2.1) we have

$$
\begin{aligned}
\mathscr{S}^{n}\left(W^{n}\right)= & \left(W^{n+1}-2 W^{n}+W^{n-1}\right) \\
& +k^{2}\left(q_{1} L_{h}^{n+1} W^{n+1}+q_{1} L_{h}^{n-1} W^{n-1}-2 p_{1} L_{h}^{n} W^{n}\right) \\
& +k^{4}\left(q_{2}\left(L_{h}^{n+1}\right)^{2} W^{n+1}+q_{2}\left(L_{h}^{n-1}\right)^{2} W^{n-1}-2 p_{2}\left(L_{h}^{n}\right)^{2} W^{n}\right) \\
& -k^{4}\left(q_{1}-\frac{1}{12}\right)\left\{L_{h}^{(2) n} W^{n}+2 L_{h}^{(1) n}\left[\frac{1}{k}\left(W^{n}-W^{n-1}\right)-\frac{k}{2} L_{h}^{n} W^{n}\right]\right\}, \\
& 1 \leqslant n \leqslant J-1 .
\end{aligned}
$$

Now, for $v:\left[0, t^{*}\right] \rightarrow H^{j}, j \geqslant 0$, it follows that

$$
\begin{aligned}
\left\|v^{n+1}-2 v^{n}+v^{n-1}\right\|_{j} & =\left\|\int_{-k}^{k}(k-|\tau|) v^{(2)}\left(t_{n}+\tau\right) d \tau\right\|_{j} \\
& \leqslant k^{2} \sup _{t^{n-1} \leqslant \tau \leqslant t^{n+1}}\left\|v^{(2)}(\tau)\right\|_{j} .
\end{aligned}
$$

It follows then from (2.10) and (2.3) for $1 \leqslant n \leqslant J-1, \phi \in S_{h}$, that

$$
\begin{aligned}
& \left(W^{n+1}-2 W^{n}+W^{n-1}, \phi\right) \\
& =\left(W^{n+1}-u^{n+1}-2\left(W^{n}-u^{n}\right)+W^{n-1}-u^{n-1}, \phi\right) \\
& \quad+\left(u^{n+1}-2 u^{n}+u^{n-1}, \phi\right) \\
& \leqslant\left(u^{n+1}-2 u^{n}+u^{n-1}, \phi\right)+C k^{2} h^{r}\left(\left\|u^{0}\right\|_{r+2}+\left\|u_{t}^{0}\right\|_{r+1}\right)\|\phi\| .
\end{aligned}
$$

Since $L_{h} W=-P u^{(2)}$ we have, for $\phi \in S_{h}, 1 \leqslant n \leqslant J-1$,

$$
\begin{gathered}
\left(q_{1} L_{h}^{n+1} W^{n+1}+q_{1} L_{h}^{n-1} W^{n-1}-2 p_{1} L_{h}^{n} W^{n}, \phi\right) \\
=-\left(q_{1} u^{(2) n+1}+q_{1} u^{(2) n-1}-2 p_{1} u^{(2) n}, \phi\right) .
\end{gathered}
$$

The analog of (1.20) for $u, L$ and (1.16) yield now, for $1 \leqslant n \leqslant J-1$,

$$
\begin{aligned}
& q_{2}\left(L_{h}^{n+1}\right)^{2} W^{n+1}+q_{2}\left(L_{h}^{n-1}\right)^{2} W^{n-1}-2 p_{2}\left(L_{h}^{n}\right)^{2} W^{n} \\
& \quad-\left(q_{1}-\frac{1}{12}\right)\left\{L_{h}^{(2) n} W^{n}+2 L_{h}^{(1) n}\left[\frac{1}{k}\left(W^{n}-W^{n-1}\right)-\frac{k}{2} L_{h}^{n} W^{n}\right]\right\} \\
&= P\left(q_{2} u^{(4) n+1}+q_{2} u^{(4) n-1}-2 p_{2} u^{(4) n}\right) \\
&+q_{2}\left[\left(L_{h}^{n+1}\right)^{2} W^{n+1}-P\left(L^{n+1}\right)^{2} u^{n+1}\right] \\
&+q_{2}\left[\left(L_{h}^{n-1}\right)^{2} W^{n-1}-P\left(L^{n-1}\right)^{2} u^{n-1}\right] \\
&-2 p_{2}\left[\left(L_{h}^{n}\right)^{2} W^{n}-P\left(L^{n}\right)^{2} u^{n}\right]-\left(q_{1}-\frac{1}{12}\right)\left(L_{h}^{(2) n} W^{n}-P L^{(2) n} u^{n}\right) \\
&-2\left(q_{1}-\frac{1}{12}\right)\left\{L_{h}^{(1) n}\left[\frac{1}{k}\left(W^{n}-W^{n-1}\right)-\frac{k}{2} L_{h}^{n} W^{n}\right]-P L^{(1) n} u^{(1) n}\right\} \\
&+q_{2} P\left[\left(L^{(2) n+1} u^{n+1}+L^{(2) n-1} u^{n-1}-2 L^{(2) n} u^{n}\right)\right. \\
&\left.+2\left(L^{(1) n+1} u^{(1) n+1}+L^{(1) n-1} u^{(1) n-1}-2 L^{(1) n} u^{(1) n}\right)\right] .
\end{aligned}
$$


Since $L_{h}^{2} W-P L^{2} u=-L_{h}\left(P-P_{I}\right) u^{(2)}$, we have, using (2.2), (1.2), (1.10) that for $1 \leqslant n \leqslant J-1, \phi \in S_{h}$,

$$
\begin{gathered}
\mid\left(q_{2}\left[\left(L_{h}^{n+1}\right)^{2} W^{n+1}-P\left(L^{n+1}\right)^{2} u^{n+1}\right]\right. \\
+q_{2}\left[\left(L_{h}^{n-1}\right)^{2} W^{n-1}-P\left(L^{n-1}\right)^{2} u^{n-1}\right] \\
\left.\quad-2 p_{2}\left[\left(L_{h}^{n}\right)^{2} W^{n}-P\left(L^{n}\right)^{2} u^{n}\right], \phi\right) \mid \\
\leqslant C h^{r}\left(\left\|u^{0}\right\|_{r+2}+\left\|u_{t}^{0}\right\|_{r+1}\right)\left\|L_{h}^{n} \phi\right\| .
\end{gathered}
$$

By (1.1), $L_{h}^{(2)} W-L^{(2)} u=-\left(L_{h}^{(2)} T_{h}-L^{(2)} T\right) u^{(2)}$. Then, Lemma 2.2 and (1.2) give for $\phi \in S_{h}, 0 \leqslant n \leqslant J$,

$$
\left|\left(L_{h}^{(2) n} W^{n}-P L^{(2) n} u^{n}, \phi\right)\right| \leqslant C h^{r}\left(\left\|u^{0}\right\|_{r}+\left\|u_{t}^{0}\right\|_{r-1}\right)\left\|L_{h}^{n} \phi\right\| .
$$

We also obtain for $1 \leqslant n \leqslant J$,

$$
\begin{aligned}
L_{h}^{(1) n}[ & \left.\frac{1}{k}\left(W^{n}-W^{n-1}\right)-\frac{k}{2} L_{h}^{n} W^{n}\right]-P L^{(1) n} u^{(1) n} \\
= & L_{h}^{(1) n} P\left[\frac{1}{k} \int_{t^{n-1}}^{t^{n}}\left(W^{(1)}(\tau)-u^{(1)}(\tau)\right) d \tau\right]+L_{h}^{(1) n} P z^{n} \\
& +L_{h}^{(1) n} P u^{(1) n}-P L^{(1) n} u^{(1) n}
\end{aligned}
$$

where

$$
z^{n} \equiv \frac{1}{k}\left(u^{n}-u^{n-1}\right)-u^{(1) n}+\frac{k}{2} u^{(2) n}
$$

Now (1.8) and (2.3) give, for $\phi \in S_{h}, 1 \leqslant n \leqslant J$, that

$$
\begin{aligned}
& \left|\left(L_{h}^{(1) n} P\left[\frac{1}{k} \int_{t^{n-1}}^{t^{n}}\left(W^{(1)}(\tau)-u^{(1)}(\tau)\right) d \tau\right], \phi\right)\right| \\
& \quad \leqslant\left\|T_{h}^{n} L_{h}^{(1) n} P\right\| \sup _{t^{n-1} \leqslant \tau \leqslant t^{n}}\left\|W^{(1)}(\tau)-u^{(1)}(\tau)\right\|\left\|L_{h}^{n} \phi\right\| \\
& \quad \leqslant C h^{r}\left(\left\|u^{0}\right\|_{r+1}+\left\|u_{t}^{0}\right\|_{r}\right)\left\|L_{h}^{n} \phi\right\| .
\end{aligned}
$$

Since Taylor's theorem and (2.17) give for $i=2,3, m \geqslant 0,1 \leqslant n \leqslant J$, that

$$
\left\|z^{n}\right\|_{m} \leqslant C(i) k^{i-1} \sup _{t^{n-1} \leqslant \tau \leqslant t^{n}}\left\|u^{(i)}(\tau)\right\|_{m}
$$

we obtain by (1.8), (1.2), (2.4) for $\phi \in S_{h}, 1 \leqslant n \leqslant J$, that

$$
\begin{aligned}
\left|\left(L_{h}^{(1) n} P z^{n}, \phi\right)\right| & =\left|\left(L_{h}^{(1) n}\left[T_{h}^{n} L^{n}+\left(P-P_{I}^{n}\right)\right] z^{n}, \phi\right)\right| \\
& \leqslant\left\|L_{h}^{(1) n} T_{h}^{n}\right\|\left\|L^{n} z^{n}\right\|\|\phi\|+\left\|T_{h}^{n} L_{h}^{(1) n} P\right\|\left\|\left(P-P_{I}^{n}\right) z^{n}\right\|\left\|L_{h}^{n} \phi\right\| \\
& \leqslant C k^{2}\left(\left\|u^{0}\right\|_{5}+\left\|u_{t}^{0}\right\|_{4}\right)\|\phi\|+C k h^{r}\left(\left\|u^{0}\right\|_{r+2}+\left\|u_{t}^{0}\right\|_{r+1}\right)\left\|L_{h}^{n} \phi\right\| .
\end{aligned}
$$

Now, since on $D_{L}, L_{h}^{(1)} P-P L^{(1)}=L_{h}^{(1)}\left(P-P_{I}\right)+\left(L_{h}^{(1)} T_{h}-P L^{(1)} T\right) L$, it follows by Lemma 2.2, (1.8), (2.2), (1.2), for $\phi \in S_{h}, 0 \leqslant n \leqslant J$,

$$
\left|\left(L_{h}^{(1) n} P u^{(1) n}-P L^{(1) n} u^{(1) n}, \phi\right)\right| \leqslant C h^{r}\left(\left\|u^{0}\right\|_{r+1}+\left\|u_{t}^{0}\right\|_{r}\right)\left\|L_{h}^{n} \phi\right\| .
$$

Finally, by (2.10), (1.2) it is seen that, for $\phi \in S_{h}, 1 \leqslant n \leqslant J-1$,

$$
\begin{aligned}
& \mid\left(L^{(2) n+1} u^{n+1}+L^{(2) n-1} u^{n-1}-2 L^{(2) n} u^{n}\right. \\
& \left.\quad+2\left[L^{(1) n+1} u^{(1) n+1}+L^{(1) n-1} u^{(1) n-1}-2 L^{(1) n} u^{(1) n}\right], \phi\right) \mid \\
& \leqslant C k^{2}\left(\left\|u^{0}\right\|_{5}+\left\|u_{t}^{0}\right\|_{4}\right)\|\phi\| .
\end{aligned}
$$


Hence, by (2.9) through (2.21) it follows that for $\phi \in S_{h}, 1 \leqslant n \leqslant J-1$,

$$
\begin{aligned}
\left|\left(\mathscr{S}^{n}\left(W^{n}\right), \phi\right)\right| \leqslant & \left|\left(q\left(-k^{2} D_{t}^{2}\right)\left(u^{n+1}+u^{n-1}\right)-2 p\left(-k^{2} D_{t}^{2}\right) u^{n}, \phi\right)\right| \\
& +C k^{2} h^{r}\left(\left\|u^{0}\right\|_{r+2}+\left\|u_{t}^{0}\right\|_{r+1}\right)\left(\|\phi\|+\left(\left|p_{2}\right|+\left|q_{2}\right|\right) k^{2}\left\|L_{h}^{n} \phi\right\|\right) \\
& +C\left|q_{1}-\frac{1}{12}\right| k^{2}\left\{h^{r}\left(\left\|u^{0}\right\|_{r+2}+\left\|u_{t}^{0}\right\|_{r+1}\right) k^{2}\left\|L_{h}^{n} \phi\right\|\right. \\
& \left.+k^{4}\left(\left\|u^{0}\right\|_{5}+\left\|u_{t}^{0}\right\|_{4}\right)\|\phi\|\right\} \\
& +C\left|q_{2}\right| k^{6}\left(\left\|u^{0}\right\|_{5}+\left\|u_{t}^{0}\right\|_{4}\right)\|\phi\| .
\end{aligned}
$$

(2.8) now follows from (2.6).

Remark. (2.22) was written to encompass all fourth-order schemes including the Størmer-Numerov method, which corresponds to $p_{2}=q_{2}=0, q_{1}=1 / 12, p_{1}=$ $-5 / 12$ and therefore has no $\left\|L_{h}^{n} \phi\right\|$ term in the right-hand side of (2.8).

We now prove the main result of this section, namely a bound on $E^{n}=U^{n}-W^{n}$, obtained by the energy method.

THEOREM 2.1. Let $U^{0}, U^{1}$ be given elements of $S_{h}$ and let $\left\{U^{n}\right\}_{n=2}^{J}$ be the solution of (2.1). Let $\left(q_{1}, q_{2}\right) \in \overline{\mathscr{R}}$ and if $\left(q_{1}, q_{2}\right) \in \partial \mathscr{R}$ assume in addition that

(2.23) $k h^{-1} \leqslant \lambda$, for any constant $\lambda>0$, if $\left(q_{1}, q_{2}\right) \in \partial \mathscr{R}_{1} \cup \partial \mathscr{R}_{2} \cup\{A\}$, and

(2.24) $k h^{-1}<\left[C_{I}\left(\frac{1}{4}-q_{1}\right)\right]^{-1 / 2}$, with $C_{I}$ as in $(1.7)$, if $\left(q_{1}, q_{2}\right) \in \partial \mathscr{R}_{3} \cup\{B\}$.

Then, for compatible $u^{0} \in H^{\mu}, u_{t}^{0} \in H^{\mu-1}$ with $\mu=\max (6, r+2)$ and for $k$ sufficiently small, there exists a constant $C$, independent of $h, k$, and $u$, such that for $0 \leqslant n \leqslant J$

$$
\begin{aligned}
\left\|E^{n}\right\| \leqslant & \left\|E^{0}\right\|+C k^{-1}\left|e^{0.1}\right|^{1 / 2} \\
& +C\left\{k^{4}\left(\left\|u^{0}\right\|_{6}+\left\|u_{t}^{0}\right\|_{5}\right)+h^{r}\left(\left\|u^{0}\right\|_{r+2}+\left\|u_{t}^{0}\right\|_{r+1}\right)\right\}
\end{aligned}
$$

where

$$
\begin{aligned}
e^{0.1} \equiv & \left\|E^{1}-E^{0}\right\|^{2}+k^{2}\left(\frac{q_{1}-p_{1}}{2}\right)\left(L_{h}^{1}\left(E^{1}+E^{0}\right), E^{1}+E^{0}\right) \\
& +k^{2}\left(\frac{q_{1}+p_{1}}{2}\right)\left(L_{h}^{1}\left(E^{1}-E^{0}\right), E^{1}-E^{0}\right) \\
& +k^{4}\left(\frac{q_{2}-p_{2}}{2}\right)\left\|L_{h}^{1}\left(E^{1}+E^{0}\right)\right\|^{2}+k^{4}\left(\frac{q_{2}+p_{2}}{2}\right)\left\|L_{h}^{1}\left(E^{1}-E^{0}\right)\right\|^{2} .
\end{aligned}
$$

Proof. Taking the $L^{2}$-inner product of both sides of (2.5) with $E^{n+1}-E^{n-1}$ for $1 \leqslant n \leqslant J-1$ and using the symmetry of $Q^{j}, P^{j}$ we obtain

$$
\begin{gathered}
\left(Q^{n+1} E^{n+1}, E^{n+1}\right)-\left(Q^{n-1} E^{n-1}, E^{n-1}\right)-\left(\left(Q^{n+1}-Q^{n-1}\right) E^{n+1}, E^{n-1}\right) \\
-2\left[\left(P^{n+1} E^{n+1}, E^{n}\right)-\left(P^{n} E^{n}, E^{n-1}\right)\right]+2\left(\left(P^{n+1}-P^{n}\right) E^{n+1}, E^{n}\right) \\
=\left(\Lambda^{n}, E^{n+1}-E^{n-1}\right)-\left(\mathscr{S}^{n}\left(W^{n}\right), E^{n+1}-E^{n-1}\right), \quad 1 \leqslant n \leqslant J-1,
\end{gathered}
$$

where, for $1 \leqslant n \leqslant J-1$,

$$
\Lambda^{n} \equiv\left(q_{1}-\frac{1}{12}\right) k^{4}\left\{L_{h}^{(2) n} E^{n}+2 L_{h}^{(1) n}\left[\frac{1}{k}\left(E^{n}-E^{n-1}\right)-\frac{k}{2} L_{h}^{n} E^{n}\right]\right\}
$$


Summing (2.27) from $n=1$ to $n=l, 1 \leqslant l \leqslant J-1$ and rearranging, yields

$$
\begin{aligned}
\frac{1}{2}\left(\left(Q^{l+1}-P^{l+1}\right)\left(E^{l+1}+E^{l}\right), E^{l+1}+E^{l}\right) \\
\quad+\frac{1}{2}\left(\left(Q^{l+1}+P^{l+1}\right)\left(E^{l+1}-E^{l}\right), E^{l+1}-E^{l}\right) \\
=\frac{1}{2}\left(\left(Q^{1}-P^{1}\right)\left(E^{1}+E^{0}\right), E^{1}+E^{0}\right) \\
\quad+\frac{1}{2}\left(\left(Q^{1}+P^{1}\right)\left(E^{1}-E^{0}\right), E^{1}-E^{0}\right)-\left(\left(Q^{1}-Q^{0}\right) E^{0}, E^{0}\right) \\
\quad+\left(\left(Q^{l+1}-Q^{l}\right) E^{l}, E^{l}\right)+\sum_{n=1}^{l}\left(\left(Q^{n+1}-Q^{n-1}\right) E^{n+1}, E^{n-1}\right) \\
\quad-2 \sum_{n=1}^{l}\left(\left(P^{n+1}-P^{n}\right) E^{n+1}, E^{n}\right)+\sum_{n=1}^{l}\left(\Lambda^{n}, E^{n+1}-E^{n-1}\right) \\
\quad-\sum_{n=1}^{l}\left(\mathscr{S}^{n}\left(W^{n}\right), E^{n+1}-E^{n-1}\right),
\end{aligned}
$$

which, upon expanding the polynomials $Q^{n}, P^{n}$ and using (2.26), may be written as

$$
\begin{aligned}
\| E^{\prime+1} & -E^{\prime} \|^{2}+k^{2}\left(\frac{q_{1}-p_{1}}{2}\right)\left(L_{h}^{l+1}\left(E^{l+1}+E^{l}\right), E^{l+1}+E^{l}\right) \\
& +k^{2}\left(\frac{q_{1}+p_{1}}{2}\right)\left(L_{h}^{l+1}\left(E^{l+1}-E^{l}\right), E^{l+1}-E^{l}\right) \\
& +k^{4}\left(\frac{q_{2}-p_{2}}{2}\right)\left\|L_{h}^{l+1}\left(E^{l+1}+E^{\prime}\right)\right\|^{2}+k^{4}\left(\frac{q_{2}+p_{2}}{2}\right)\left\|L_{h}^{l+1}\left(E^{l+1}-E^{l}\right)\right\|^{2} \\
= & e^{0,1}-q_{1} k^{2}\left(\left(L_{h}^{1}-L_{h}^{0}\right) E^{0}, E^{0}\right)-q_{2} k^{4}\left(\left[\left(L_{h}^{1}\right)^{2}-\left(L_{h}^{0}\right)^{2}\right] E^{0}, E^{0}\right) \\
& +q_{1} k^{2}\left(\left(L_{h}^{l+1}-L_{h}^{l}\right) E^{l}, E^{l}\right)+q_{2} k^{4}\left(\left[\left(L_{h}^{l+1}\right)^{2}-\left(L_{h}^{l}\right)^{2}\right] E^{\prime}, E^{\prime}\right) \\
& +q_{1} k^{2} \sum_{n=1}^{l}\left(\left(L_{h}^{n+1}-L_{h}^{n-1}\right) E^{n+1}, E^{n-1}\right) \\
& -2 p_{1} k^{2} \sum_{n=1}^{l}\left(\left(L_{h}^{n+1}-L_{h}^{n}\right) E^{n+1}, E^{n}\right) \\
& +q_{2} k^{4} \sum_{n=1}^{l}\left(\left[\left(L_{h}^{n+1}\right)^{2}-\left(L_{h}^{n-1}\right)^{2}\right] E^{n+1}, E^{n-1}\right) \\
& -2 p_{2} k^{4} \sum_{n=1}^{l}\left(\left[\left(L_{h}^{n+1}\right)^{2}-\left(L_{h}^{n}\right)^{2}\right] E^{n+1}, E^{n}\right) \\
& +\sum_{n=1}^{l}\left(\Lambda^{n}, E^{n+1}-E^{n-1}\right) \\
& -\sum_{n=1}^{l}\left(\mathscr{S}^{n}\left(W^{n}\right), E^{n+1}-E^{n-1}\right), \\
& 1 \leqslant l \leqslant J-1 . \\
&
\end{aligned}
$$

From (1.5), (1.6) it follows that for $0 \leqslant m, n, i, j \leqslant J, \phi, \psi \in S_{h}$ :

$$
\left|\left(\left(L_{h}^{m}-L_{h}^{n}\right) \phi, \psi\right)\right| \leqslant C|m-n| k\left(L_{h}^{i} \phi, \phi\right)^{1 / 2}\left(L_{h}^{j} \psi, \psi\right)^{1 / 2}
$$


Hence, for $1 \leqslant l \leqslant J-1,(2.30)$ and the arithmetic-geometric mean inequality yield

$$
\begin{aligned}
& -q_{1} k^{2}\left(\left(L_{h}^{1}-L_{h}^{0}\right) E^{0}, E^{0}\right)+q_{1} k^{2}\left(\left(L_{h}^{l+1}-L_{h}^{l}\right) E^{l}, E^{l}\right) \\
& \quad+q_{1} k^{2} \sum_{n=1}^{l}\left(\left(L_{h}^{n+1}-L_{h}^{n-1}\right) E^{n+1}, E^{n-1}\right) \\
& \quad-2 p_{1} k^{2} \sum_{n=1}^{l}\left(\left(L_{h}^{n+1}-L_{h}^{n}\right) E^{n+1}, E^{n}\right) \\
& \leqslant C k^{3} \sum_{n=0}^{l}\left[\left(L_{h}^{n+1} E^{n+1}, E^{n+1}\right)+\left(L_{h}^{n+1} E^{n}, E^{n}\right)\right] \\
& \leqslant C k \sum_{n=0}^{l} k^{2}\left[\left(L_{h}^{n+1}\left(E^{n+1}+E^{n}\right), E^{n+1}+E^{n}\right)\right. \\
& \left.\quad+\left(L_{h}^{n+1}\left(E^{n+1}-E^{n}\right), E^{n+1}-E^{n}\right)\right] .
\end{aligned}
$$

Also, for $0 \leqslant m, n, i, j \leqslant J$ and $\phi, \psi \in S_{h}$, it follows, as in (4.6) of [7], by (1.8) and (1.10) that

$$
\left|\left(\left[\left(L_{h}^{m}\right)^{2}-\left(L_{h}^{n}\right)^{2}\right] \phi, \psi\right)\right| \leqslant C|m-n| k\left\|L_{h}^{i} \phi\right\|\left\|L_{h}^{j} \psi\right\| .
$$

Hence, (2.32) and the arithmetic-geometric mean inequality give

$$
\begin{aligned}
-q_{2} k^{4}( & {\left.\left[\left(L_{h}^{1}\right)^{2}-\left(L_{h}^{0}\right)^{2}\right] E^{0}, E^{0}\right)+q_{2} k^{4}\left(\left[\left(L_{h}^{l+1}\right)^{2}-\left(L_{h}^{l}\right)^{2}\right] E^{l}, E^{\prime}\right) } \\
& +q_{2} k^{4} \sum_{n=1}^{l}\left(\left[\left(L_{h}^{n+1}\right)^{2}-\left(L_{h}^{n-1}\right)^{2}\right] E^{n+1}, E^{n-1}\right) \\
& -2 p_{2} k^{4} \sum_{n=1}^{l}\left(\left[\left(L_{h}^{n+1}\right)^{2}-\left(L_{h}^{n}\right)^{2}\right] E^{n+1}, E^{n}\right) \\
\leqslant & C k^{5} \sum_{n=0}^{l}\left(\left\|L_{h}^{n+1} E^{n+1}\right\|^{2}+\left\|L_{h}^{n+1} E^{n}\right\|^{2}\right) \\
\leqslant & C k \sum_{n=0}^{l} k^{4}\left(\left\|L_{h}^{n+1}\left(E^{n+1}+E^{n}\right)\right\|^{2}+\left\|L_{h}^{n+1}\left(E^{n+1}-E^{n}\right)\right\|^{2}\right) .
\end{aligned}
$$

Also, by (2.28), (1.5), (1.6), (1.8), (1.10) we obtain for $1 \leqslant l \leqslant J-1$,

$$
\begin{aligned}
& \sum_{n=1}^{l}\left(\Lambda^{n}, E^{n+1}-E^{n-1}\right) \\
& \leqslant C k^{4} \sum_{n=0}^{l}\left[\left(L_{h}^{n+1} E^{n}, E^{n}\right)+\left(L_{h}^{n+1} E^{n+1}, E^{n+1}\right)\right] \\
& \quad+C k^{3} \sum_{n=0}^{l}\left[\left(L_{h}^{n+1} E^{n}, E^{n}\right)+\left(L_{h}^{n+1} E^{n+1}, E^{n+1}\right)\right] \\
& \quad+C k^{5} \sum_{n=1}^{l}\left\|L_{h}^{n} E^{n}\right\|\left\|T_{h}^{n} L_{h}^{(1) n} P\right\|\left\|L_{h}^{n}\left(E^{n+1}-E^{n-1}\right)\right\| \\
& \leqslant \\
& \quad C k \sum_{n=0}^{l} k^{2}\left[\left(L_{h}^{n+1}\left(E^{n+1}+E^{n}\right), E^{n+1}+E^{n}\right)\right. \\
& \quad+C k \sum_{n=0}^{l} k^{4}\left(\left\|L_{h}^{n+1}\left(E^{n+1}+E^{n}\right)\right\|^{2}+\left\|L_{h}^{n+1}\left(E^{n+1}-E^{n}\right)\right\|^{2}\right)
\end{aligned}
$$


Finally, defining

$$
\sigma_{k, h} \equiv k^{4}\left(\left\|u^{0}\right\|_{6}+\left\|u_{t}^{0}\right\|_{5}\right)+h^{r}\left(\left\|u^{0}\right\|_{r+2}+\left\|u_{t}^{0}\right\|_{r+1}\right),
$$

we have, by (2.8), (1.10) and the arithmetic-geometric mean inequality

$$
\begin{aligned}
& -\sum_{n=1}^{l}\left(\mathscr{S}^{n}\left(W^{n}\right), E^{n+1}-E^{n-1}\right) \\
& \leqslant \sum_{n=1}^{l} C k^{2} \sigma_{k, h}\left(\left\|E^{n+1}-E^{n-1}\right\|+k^{2}\left\|L_{h}^{n+1}\left(E^{n+1}-E^{n-1}\right)\right\|\right) \\
& \leqslant C k^{2} \sigma_{k, h}^{2}+C k \sum_{n=0}^{l}\left\|E^{n+1}-E^{n}\right\|^{2}+C k \sum_{n=0}^{l} k^{4}\left\|L_{h}^{n+1}\left(E^{n+1}-E^{n}\right)\right\|^{2}, \\
& \quad 1 \leqslant l \leqslant J-1 .
\end{aligned}
$$

Therefore, it follows from (2.29), (2.31), (2.33), (2.34) and (2.36) that for $0 \leqslant l \leqslant$ $J-1$

$$
\begin{aligned}
\| E^{l+1}- & E^{\prime} \|^{2}+k^{2}\left(\frac{q_{1}-p_{1}}{2}\right)\left(L_{h}^{l+1}\left(E^{l+1}+E^{l}\right), E^{l+1}+E^{l}\right) \\
& +k^{2}\left(\frac{q_{1}+p_{1}}{2}\right)\left(L_{h}^{l+1}\left(E^{l+1}-E^{l}\right), E^{l+1}-E^{l}\right) \\
& +k^{4}\left(\frac{q_{2}-p_{2}}{2}\right)\left\|L_{h}^{l+1}\left(E^{l+1}+E^{l}\right)\right\|^{2}+k^{4}\left(\frac{q_{2}+p_{2}}{2}\right)\left\|L_{h}^{l+1}\left(E^{l+1}-E^{l}\right)\right\|^{2} \\
(2.37) \leqslant & e^{0.1}+C k^{2} \sigma_{k, h}^{2}+C k \sum_{n=0}^{l}\left\|E^{n+1}-E^{n}\right\|^{2} \\
& +C k \sum_{n=0}^{l} k^{2}\left[\left(L_{h}^{n+1}\left(E^{n+1}+E^{n}\right), E^{n+1}+E^{n}\right)\right. \\
& \left.+\left(L_{h}^{n+1}\left(E^{n+1}-E^{n}\right), E^{n+1}-E^{n}\right)\right] \\
& +C k \sum_{n=0}^{l} k^{4}\left(\left\|L_{h}^{n+1}\left(E^{n+1}+E^{n}\right)\right\|^{2}+\left\|L_{h}^{n+1}\left(E^{n+1}-E^{n}\right)\right\|^{2}\right) .
\end{aligned}
$$

We now embark upon the stability investigation. We distinguish a number of cases depending on the position of $\left(q_{1}, q_{2}\right)$ in $\overline{\mathscr{R}}$.

I.i: $\left(q_{1}, q_{2}\right) \in \mathscr{R}, q_{1}>1 / 4$. In this case by (1.15), $q_{1}+p_{1}>0$ and by (1.18) we see that the coefficients of all terms in the left-hand side of (2.37) are positive. Applying (the discrete) Gronwall's lemma to (2.37) yields, for $k$ sufficiently small,

$$
\left\|E^{n+1}-E^{n}\right\|^{2} \leqslant C\left(e^{0,1}+k^{2} \sigma_{k, h}^{2}\right), \quad 0 \leqslant n \leqslant J-1 .
$$

Taking square roots in (2.38) and using the fact that

$$
\left\|E^{n+1}\right\| \leqslant \sum_{j=0}^{n}\left\|E^{j+1}-E^{j}\right\|+\left\|E^{0}\right\|, \quad \text { for } 0 \leqslant n \leqslant J-1,
$$

we obtain (2.25).

I.ii: $\left(q_{1}, q_{2}\right) \in \mathscr{R}, q_{1}=1 / 4$. Now, since $q_{1}+p_{1}=0$, the third term in the left-hand side of (2.37) is missing. For the analogous term in the right-hand side of 
(2.37) the Cauchy-Schwarz and the arithmetic-geometric mean inequalities yield, for $0 \leqslant l \leqslant J-1$,

$$
\begin{aligned}
& C k \sum_{n=0}^{l} k^{2}\left(L_{h}^{n+1}\left(E^{n+1}-E^{n}\right), E^{n+1}-E^{n}\right) \\
& \quad \leqslant C k \sum_{n=0}^{l}\left\{k^{4}\left\|L_{h}^{n+1}\left(E^{n+1}-E^{n}\right)\right\|^{2}+\left\|E^{n+1}-E^{n}\right\|^{2}\right\} .
\end{aligned}
$$

Gronwall's lemma may now be applied to (2.37) and (2.25) follows as above.

I.iii: $\left(q_{1}, q_{2}\right) \in \mathscr{R}, q_{1}<1 / 4$. In this case all terms in the left-hand side of (2.37) have positive coefficients except the third, since $q_{1}+p_{1}<0$. However, the CauchySchwarz and the arithmetic-geometric mean inequalities yield, for any $\varepsilon>0$

$$
\begin{aligned}
& k^{2}\left(L_{h}^{l+1}\left(E^{l+1}-E^{l}\right), E^{l+1}-E^{l}\right) \\
& \quad \leqslant \frac{k^{4}}{2 \varepsilon}\left\|L_{h}^{l+1}\left(E^{l+1}-E^{l}\right)\right\|^{2}+\frac{\varepsilon}{2}\left\|E^{l+1}-E^{l}\right\|^{2} .
\end{aligned}
$$

It follows that the left-hand side of $(2.37)$ is bounded below by

$$
\begin{aligned}
& {\left[1+\frac{\left(q_{1}+p_{1}\right) \varepsilon}{4}\right]\left\|E^{l+1}-E^{\prime}\right\|^{2}} \\
& +k^{2}\left(\frac{q_{1}-p_{1}}{2}\right)\left(L_{h}^{l+1}\left(E^{l+1}+E^{l}\right), E^{l+1}+E^{l}\right) \\
& +k^{4}\left(\frac{q_{2}-p_{2}}{2}\right)\left\|L_{h}^{l+1}\left(E^{l+1}+E^{l}\right)\right\|^{2} \\
& +k^{4}\left[\frac{q_{2}+p_{2}}{2}+\frac{\left(q_{1}+p_{1}\right)}{4 \varepsilon}\right] \cdot\left\|L_{h}^{l+1}\left(E^{l+1}-E^{l}\right)\right\|^{2}
\end{aligned}
$$

Both the coefficients of the first and fourth term of (2.41) will be positive iff there exists an $\varepsilon>0$ such that $\varepsilon<-4 /\left(q_{1}+p_{1}\right)$ and $\varepsilon>-\left(q_{1}+p_{1}\right) / 2\left(q_{2}+p_{2}\right)$, i.e. iff $\left(q_{1}+p_{1}\right)^{2}<8\left(q_{2}+p_{2}\right)$. This holds in the subset of $\mathscr{R}$ under consideration since the equation of the parabolic arc $\partial \mathscr{R}_{3}$ is $8\left(q_{2}+p_{2}\right)=\left(q_{1}+p_{1}\right)^{2}$. Therefore there exists a suitable $\varepsilon>0$ such that the coefficients of all terms in (2.41) are positive. After an estimate like (2.39) in the right-hand side of (2.37) we may apply Gronwall's lemma and (2.25) follows.

II.i: $\left(q_{1}, q_{2}\right) \in \partial \mathscr{R}_{1}$. In this case $q_{2}+p_{2}=0$ from (1.18) and all terms in the left-hand side of (2.37) have positive coefficients except the last one, which is missing. For the corresponding term in the right-hand side of $(2.37)$ we have, by (1.9), (2.23),

$$
C k \sum_{n=0}^{l} k^{4}\left\|L_{h}^{n+1}\left(E^{n+1}-E^{n}\right)\right\|^{2} \leqslant C \lambda^{4} k \sum_{n=0}^{l}\left\|E^{n+1}-E^{n}\right\|^{2} .
$$

Hence, the application of Gronwall's lemma is again possible and (2.25) follows.

II.ii: $\left(q_{1}, q_{2}\right)=(1 / 4,1 / 24)=A$. Now $q_{2}+p_{2}=0, q_{1}+p_{1}=0$ while $q_{2}-p_{2}>$ $0, q_{1}-p_{1}>0$. Using (1.9) and (2.23) we may estimate in the right-hand side of (2.37), in addition to (2.42),

$$
C k \sum_{n=0}^{l} k^{2}\left(L_{h}^{n+1}\left(E^{n+1}-E^{n}\right), E^{n+1}-E^{n}\right) \leqslant C \lambda^{2} k \sum_{n=0}^{l}\left\|E^{n+1}-E^{n}\right\|^{2}
$$


From (2.42) and (2.43) it follows that Gronwall's lemma may be applied again to (2.37); (2.25) follows.

III: $\left(q_{1}, q_{2}\right) \in \partial \mathscr{R}_{2}$. Now $q_{2}-p_{2}=0, q_{1}-p_{1}>0, q_{2}+p_{2}>0$ but $q_{1}+p_{1}<0$. It is still possible, since $8\left(q_{2}+p_{2}\right)>\left(q_{1}+p_{1}\right)^{2}$ on $\partial \mathscr{R}_{2}$, to repeat the analysis of case I.iii and choose $\varepsilon>0$ that enables us to hide the third term within the first and the fifth term in the left-hand side of (2.37). Using again (2.43) and the estimate

$$
\begin{aligned}
& C k \sum_{n=0}^{l} k^{4}\left\|L_{h}^{n+1}\left(E^{n+1}+E^{n}\right)\right\|^{2} \\
& \quad \leqslant C k^{5} \sum_{n=0}^{l}\left\|\left(L_{h}^{n+1}\right)^{1 / 2} P\right\|^{2}\left\|\left(L_{h}^{n+1}\right)^{1 / 2}\left(E^{n+1}+E^{n}\right)\right\|^{2} \\
& \quad \leqslant C \lambda^{2} k \sum_{n=0}^{l} k^{2}\left(L_{h}^{n+1}\left(E^{n+1}+E^{n}\right), E^{n+1}+E^{n}\right)
\end{aligned}
$$

(which follow from (1.7) and (2.23)), in the right-hand side of (2.37), we are able to complete the proof of $(2.25)$ in this case as well.

IV.i: $\left(q_{1}, q_{2}\right) \in \partial \mathscr{R}_{3}$. Now $q_{1}-p_{1}>0, q_{1}+p_{1}<0, q_{2}+p_{2}>0, q_{2}-p_{2}>0$ but we are no longer able to find $\varepsilon>0$ for which all terms of (2.41) have positive coefficients since $\left(q_{1}+p_{1}\right)^{2}=8\left(q_{2}+p_{2}\right)$ on $\partial \mathscr{R}_{3}$. However, by (1.7)

$$
\begin{gathered}
k^{2}\left(\frac{q_{1}+p_{1}}{2}\right)\left(L_{h}^{l+1}\left(E^{l+1}-E^{l}\right), E^{l+1}-E^{l}\right) \\
\geqslant C_{I}\left(\frac{q_{1}+p_{1}}{2}\right) \frac{k^{2}}{h^{2}}\left\|E^{l+1}-E^{\prime}\right\|^{2} .
\end{gathered}
$$

Hence, noting, by (1.15), that $\left(q_{1}+p_{1}\right) / 2=-\left(\frac{1}{4}-q_{1}\right)$ and using the hypothesis (2.24) we are able to hide the third term of the left-hand side of (2.37) within the first. An estimate of the form (2.43) prepares the right-hand of (2.37) for Gronwall's lemma and the results follow.

IV.ii: $\left(q_{1}, q_{2}\right)=(1 / 12,1 / 144)=B$. In this final case (which corresponds to the $r(x)$ obtained as the real part of the $(2,2)$ Padé approximant of $e^{i x}$ ) we have $q_{2}-p_{2}=0$. As in case IV.i we may hide the third term within the first in the left-hand side of (2.37). Two estimates of the form (2.43) and (2.44) make the right-hand side of (2.37) amenable to the application of Gronwall's lemma and (2.25) follows.

Remark. Obviously, if $\left(q_{1}, q_{2}\right) \notin \overline{\mathscr{R}}$ but still, e.g., $q_{1}, q_{2} \geqslant 0$, we can, by repeating appropriate parts of the above analysis handle, under the hypothesis that $k h^{-1}$ be small, all such conditionally stable cases too, i.e. cases for which $|r(x)| \leqslant 1$ only for $|x| \leqslant \alpha<\infty$.

3. Starting the Base Scheme. In this section we shall consider the problem of choosing suitable initial values, $U^{0}, U^{1}$ in $S_{h}$ for the base scheme (2.1), that make the initial error term $\left\|E^{0}\right\|+C k^{-1}\left|e^{0,1}\right|^{1 / 2}$ in (2.25) of optimal order $O\left(k^{4}+h^{r}\right)$.

Taking $U^{0}=W^{0}=T_{h}^{0} L^{0} u^{0}$ will give of course $E^{0}=0$. Hence (2.26) shows that it would suffice to choose $U^{1}$ so that

$$
\left\|E^{1}\right\|+k\left(L_{h}^{1} E^{1}, E^{1}\right)^{1 / 2}+k^{2}\left\|L_{h}^{1} E^{1}\right\|=O\left(k^{5}+k h^{r}\right) .
$$


One way to do this is to choose $U^{1}$ by performing one step of a single-step fully discrete Galerkin method for the hyperbolic equation (1.1) written in first-order system form. Such methods (corresponding to rational approximation to $e^{i x}$ ) are analyzed in [5], [6], to results of which we shall frequently refer in sequel.

The specific single-step method that we shall use corresponds to the only fourthorder accurate rational approximation to $e^{z}, z \in \mathbf{C}$, with quadratic numerator and denominator, namely to the $(2,2)$ Padé approximate of $e^{z}$, given by

$$
\tilde{r}(z)=\left(1+z / 2+z^{2} / 12\right) /\left(1-z / 2+z^{2} / 12\right),
$$

which satisfies, for $z$ sufficiently small,

$$
\left|\tilde{r}(z)-e^{z}\right| \leqslant C|z|^{5}
$$

and is, of course, $A$-stable. Here, however, we shall only need its stability on the imaginary axis, i.e., that

$$
|\tilde{r}(i x)| \leqslant 1 \text { for } x \in \mathbf{R}^{1} .
$$

Following now [5], [6], consider the product space $S_{h}^{2}=S_{h} \times S_{h}$ and the operator on $S_{h}^{2}$ defined by

$$
\mathscr{L}_{h}(t)=\left(\begin{array}{cc}
0 & I \\
-L_{h}(t) & 0
\end{array}\right) .
$$

Define for $n=0,1,2, \ldots, \mathscr{L}_{h}^{n}=\mathscr{L}_{h}\left(t^{n}\right), \mathscr{L}_{h}^{(j) n}=D_{t}^{j} \mathscr{L}_{h}\left(t^{n}\right), j \geqslant 0$, and the operators

$$
\begin{aligned}
& \tilde{\mathscr{B}}^{n}=I+\frac{k}{2} \mathscr{L}_{h}^{n}+\frac{k^{2}}{12}\left[\left(\mathscr{L}_{h}^{n}\right)^{2}+\mathscr{L}_{h}^{(1) n}\right], \\
& \tilde{\mathscr{B}}^{n}=I-\frac{k}{2} \mathscr{L}_{h}^{n}+\frac{k^{2}}{12}\left[\left(\mathscr{L}_{h}^{n}\right)^{2}+\mathscr{L}_{h}^{(1) n}\right], \\
& \mathscr{B}^{n}=\tilde{\mathscr{B}}^{n}-\frac{k^{2}}{12} \mathscr{L}_{h}^{(1) n} .
\end{aligned}
$$

It is shown in [5] that, as a consequence of the hypotheses made, e.g., in Section 1, for $k$ sufficiently small, $\tilde{\mathscr{B}}^{n}$ and $\mathscr{B}^{n}$ are invertible. With $W=P_{I} u$ denoting as in Section 2 the elliptic projection of $u$, define $\mathscr{W}^{n}=\left[W^{n}, W^{(1) n}\right]^{T} \in S_{h}^{2}$ and let $\mathscr{U}^{1}=\left[U_{1}^{1}, U_{2}^{1}\right] \in S_{h}^{2}$ be the solution of the system

$$
\tilde{\mathscr{B}}^{1} \mathscr{U}^{1}=\tilde{\mathscr{A}}^{0} \mathscr{W}^{0} .
$$

For $\Phi=\left[\phi_{1}, \phi_{2}\right]^{T} \in S_{h}^{2}$ define the norm on $S_{h}^{2}$

$$
\|\Phi\|_{n}=\left(\left\|\phi_{1}\right\|^{2}+\left(T_{h}^{n} \phi_{2}, \phi_{2}\right)\right)^{1 / 2}, \quad n=0,1,2, \ldots
$$

We are now ready to prove a result that guarantees optimal starting for the scheme (2.1).

Proposition 3.1. Let $U^{0}=W^{0}$ and if $\mathscr{U}^{1}=\left[U_{1}^{1}, U_{2}^{1}\right]^{T}$ is the solution of (3.6), take $U^{1}=U_{1}^{1}$. Then, for compatible $u^{0} \in H^{\mu}, u_{t}^{0} \in H^{\mu-1}, \mu=\max (6, r+2)$ and for $k$ sufficiently small, there exists a constant $C$, independent of $h, k$, and $u$, such that if $e^{0,1}$ is defined by (2.26),

$$
\left\|E^{0}\right\|+k^{-1}\left|e^{0.1}\right|^{1 / 2} \leqslant C\left\{k^{4}\left(\left\|u^{0}\right\|_{6}+\left\|u_{t}^{0}\right\|_{5}\right)+h^{r}\left(\left\|u^{0}\right\|_{r+2}+\left\|u_{t}^{0}\right\|_{r+1}\right)\right\} .
$$


Proof. We have already remarked that it suffices to prove an inequality of the type (3.1). It follows from Theorem 4.1 of [6] that under our hypotheses, if $\mathscr{E}^{1}=\mathscr{U}^{1}-\mathscr{W}^{1}$, then

$$
\left\|\mathscr{B}^{1} \mathscr{E}^{1}\right\|_{1} \leqslant C k \sigma_{k, h},
$$

where $\sigma_{k, h}$ has been defined by (2.35). Now, by Lemma 4.1 of [6] we have

$$
\left\|\mathscr{E}^{1}\right\|_{1}+k\left\|\mathscr{L}_{h}^{1} \mathscr{E}^{1}\right\|_{1}+k^{2}\left\|\left(\mathscr{L}_{h}^{1}\right)^{2} \mathscr{E}^{1}\right\|_{1} \leqslant C\left\|\mathscr{B}^{1} \mathscr{E}^{1}\right\|_{1} .
$$

Hence, since $E^{1}=E_{1}^{1}$, where $\mathscr{E}^{1}=\left[E_{1}^{1}, E_{2}^{1}\right]^{T},(3.7)$ and (3.10) give

$$
\left\|E^{1}\right\| \leqslant C\left\|\mathscr{B}^{1} \mathscr{E}^{1}\right\| \text {. }
$$

(3.7) also gives that

$$
\left\|\mathscr{L}_{h}^{1} \mathscr{E}^{1}\right\|_{1}^{2}=\left\|E_{2}^{1}\right\|^{2}+\left(L_{h}^{1} E_{1}^{1}, E_{1}^{1}\right),
$$

from which (and (3.10)) we obtain

$$
k\left(L_{h}^{1} E^{1}, E^{1}\right)^{1 / 2} \leqslant C\left\|\mathscr{B}^{1} \mathscr{E}^{1}\right\|_{1} .
$$

Since $\left(\mathscr{L}_{h}^{1}\right)^{2} \mathscr{E}^{1}=\left[-L_{h}^{1} E_{1}^{1},-L_{h}^{1} E_{2}^{1}\right]^{T},(3.10)$ and (3.7) yield

$$
k^{2}\left\|L_{h}^{1} E^{1}\right\| \leqslant C\left\|\mathscr{B}^{1} \mathscr{E}^{1}\right\|_{1} .
$$

Therefore, we see from (3.9)-(3.12), that an estimate of the type (3.1) holds. Since $E^{0}=0,(3.8)$ also follows.

Combining now the results of Theorem 2.1 and Proposition 3.1 and (2.3) we can state the following optimal-order convergence result.

THEOREM 3.1. Let $\left\{U^{n}\right\}_{n=2}^{J}$ be the solution of (2.1) and $U^{0}, U^{1}$ be chosen as in Proposition 3.1. Under the hypotheses of Theorem 2.1 there exists a constant $C$, independent of $h, k$, and $u$ and such that for $0 \leqslant n \leqslant J$,

$$
\left\|u^{n}-U^{n}\right\| \leqslant C\left\{k^{4}\left(\left\|u^{0}\right\|_{6}+\left\|u_{t}^{0}\right\|_{5}\right)+h^{r}\left(\left\|u^{0}\right\|_{r+2}+\left\|u_{t}^{0}\right\|_{r+1}\right)\right\} .
$$

4. Preconditioned Iterative Methods. Since the operators $Q^{n+1}$ that multiply $U^{n+1}$ in (2.1) change at each time step, we shall consider, following [9], [7], preconditioned iterative methods with suitable starting values to solve the linear systems represented by (2.1), thus avoiding the repeated work of new matrix factorizations at each time step.

We specify first desired properties of such methods relevant to our problem. Let $H$ be a finite-dimensional Hilbert space with inner product $(\cdot, \cdot)_{H}$ and norm $\|\cdot\|_{H}=$ $(\cdot, \cdot)_{H}^{1 / 2}$. Let $A$ be a positive-definite selfadjoint operator on $H$ and suppose that we want to approximate the solution of the linear system $A x=b$, with $b$ given in $H$. Suppose that there exists another positive-definite selfadjoint operator $A_{0}$ on $H$ (the "preconditioner") with the properties that systems of the form $A_{0} y=z, z \in H$ are easily solvable and that there exist constants $0<\lambda_{0} \leqslant \lambda_{1}$ such that

$$
\lambda_{0}\left(A_{0} z, z\right)_{H} \leqslant(A z, z)_{H} \leqslant \lambda_{1}\left(A_{0} z, z\right)_{H} \text { for } z \in H \text {. }
$$

Then, there are iterative methods, cf., e.g., [1], [9], [7], [6], for solving the system $A x=b$, which, given an initial guess $x^{(0)} \in H$, generate a sequence $\left\{x^{(j)}\right\}_{j \geqslant 1}$ of approximations to $x$ and have the following properties. 
(i) Calculating $x^{(p+1)}$, given $\left\{x^{(j)}\right\}_{j=0}^{p}$, only requires multiplying $A$ with vectors, solving systems involving $A_{0}$ and computing inner products and linear combinations of vectors.

(ii) There is a smooth decreasing function $\rho:(0,1] \rightarrow[0,1)$, with $\rho(1)=0$ and a constant $C$ such that

$$
\left\|A_{0}^{1 / 2}\left(x-x^{(p)}\right)\right\|_{H} \leqslant C\left[\rho\left(\lambda_{0} / \lambda_{1}\right)\right]^{p}\left\|A_{0}^{1 / 2}\left(x-x^{(0)}\right)\right\|_{H},
$$

where $\lambda_{0}, \lambda_{1}$ are the constants in (4.1). In our application we shall perform at each step $n, 1 \leqslant n \leqslant J, p_{n}$ iterations, sufficiently many so that

$$
\left\|A_{0}^{1 / 2}\left(x-x^{\left(p_{n}\right)}\right)\right\|_{H} \leqslant \beta_{n}\left\|A_{0}^{1 / 2}\left(x-x^{(0)}\right)\right\|_{H}
$$

is achieved, where $\beta_{n}>0$ are small, preassigned tolerances. In general, we shall take $\beta_{n}=O\left(k^{\nu}\right), \nu \geqslant 1$, thus requiring, in general, $p_{n}=O\left(\ln \left(k^{-1}\right)\right)$ iterations for each $n$.

To use such iterative methods to solve the linear systems associated with the base scheme (2.1), we shall identify $\left\{H,(\cdot, \cdot)_{H}\right\}$ with $\left\{S_{h},(\cdot, \cdot)\right\}$, the operator $A$ with $Q^{n+1}$, and the preconditioner $A_{0}$ with a selfadjoint positive-definite operator ${ }^{p} Q$ on $S_{h}$, such that for each $\phi \in S_{h},\left({ }^{p} Q\right)^{-1} \phi$ is easy to find and for which there exist constants $C, C^{\prime}$ such that for all $0 \leqslant n \leqslant J-1, \phi \in S_{h}$,

$$
C\left({ }^{p} Q \phi, \phi\right) \leqslant\left(Q^{n+1} \phi, \phi\right) \leqslant C^{\prime}\left({ }^{p} Q \phi, \phi\right)
$$

is satisfied. An example of such a preconditioner is provided by the following

Lemma 4.1. Assume that $q_{1}, q_{2}>0$. Then, the operator

$$
{ }^{p} Q=\left(I+\beta k^{2} L_{h}^{0}\right)^{2}, \quad \beta>0
$$

defines a suitable preconditioner for the iterative solution of the linear systems (2.1) and satisfies (4.4).

Proof. Let $\Pi(x)=1+x+x^{2}$ and $\Pi^{n}=\Pi\left(k^{2} L_{h}^{n}\right)$. Then, since $q_{1}, q_{2}, \beta>0$, there exist positive constants $C_{i}, 1 \leqslant i \leqslant 4$, such that for all $0 \leqslant n \leqslant J, \phi \in S_{h}$,

$$
\begin{aligned}
& C_{1}\left(\Pi^{n} \phi, \phi\right) \leqslant\left(Q^{n} \phi, \phi\right) \leqslant C_{2}\left(\Pi^{n} \phi, \phi\right), \\
& C_{3}\left(\Pi^{0} \phi, \phi\right) \leqslant\left({ }^{p} Q \phi, \phi\right) \leqslant C_{4}\left(\Pi^{0} \phi, \phi\right) .
\end{aligned}
$$

(4.3) now follows from the above and (1.5) and (1.10).

Now with $V^{0}, V^{1}$ given elements of $S_{h}$, let $V^{n+1}, 1 \leqslant n \leqslant J-1$, be the approximation to the exact solution $\bar{V}^{n+1}$ of

$$
\begin{aligned}
Q^{n+1} \bar{V}^{n+1}= & 2 P^{n} V^{n}-Q^{n-1} V^{n-1} \\
& +k^{4}\left(q_{1}-\frac{1}{12}\right)\left\{L_{h}^{(2) n} V^{n}+2 L_{h}^{(1) n}\left[\frac{1}{k}\left(V^{n}-V^{n-1}\right)-\frac{k}{2} L_{h}^{n} V^{n}\right]\right\},
\end{aligned}
$$

obtained by applying $p_{n+1}$ steps of an iterative method, such as the ones described above, with a preconditioner ${ }^{p} Q$ that satisfies (4.4), so that, for $1 \leqslant n \leqslant J-1$, and a preassigned tolerance $\beta_{n+1}$, we have

$$
\left\|\left({ }^{p} Q\right)^{1 / 2}\left(\bar{V}^{n+1}-V^{n+1}\right)\right\| \leqslant \beta_{n+1}\left\|\left({ }^{p} Q\right)^{1 / 2}\left(\bar{V}^{n+1}-V_{0}^{n+1}\right)\right\| .
$$

Here $V_{0}^{n+1}$ is the initial guess which should be chosen sufficiently close to $\bar{V}^{n+1}$ so that the number of iterations to achieve (4.7) is reduced. Since $V^{n}$ is intended to 
approximate $u^{n}$ and $u(t)$ is smooth, we shall construct $V_{0}^{n+1}$ by extrapolating from previous values of $V^{n}$. Specifically, we take

$$
V_{0}^{n+1}=\left\{\begin{array}{l}
\Gamma_{n+1}^{(1)}(V) \equiv V^{n} \quad \text { for } 2 \leqslant n+1 \leqslant 4 \\
\Gamma_{n+1}^{(5)}(V) \equiv 5 V^{n}-10 V^{n-1}+10 V^{n-2}-5 V^{n-3}+V^{n-4}
\end{array}\right.
$$

for $5 \leqslant n+1 \leqslant J$.

The next result shows that such an iterative procedure, with suitable tolerances $\beta_{n+1}$ (which are in general of $O\left(k^{\nu}\right), \nu \geqslant 1$ so that $p_{n+1}=O\left(\ln \left(k^{-1}\right)\right)$ ), preserves the accuracy and stability of the base scheme. In sequel we shall put $Z^{n} \equiv V^{n}-W^{n}$, $0 \leqslant n \leqslant J$.

Proposition 4.1. Let $V^{0}, V^{1}$ be given in $S_{h}$. For $1 \leqslant n \leqslant J-1$, compute $V^{n+1}$, an approximation to the solution $\bar{V}^{n+1}$ of (4.6), by a preconditioned iterative method that satisfies properties (i), (ii) above when $\left\{H,(\cdot, \cdot)_{H}\right\}=\left\{S_{h},(\cdot, \cdot)\right\}$ with a preconditioner ${ }^{p} Q$ that satisfies (4.4). Then, there is $a \gamma>0$ such that if we use

(a) $V_{0}^{n+1}=\Gamma_{n+1}^{(1)}(V)$, given by (4.8), as an initial guess for $V^{n+1}$ and a corresponding tolerance $\beta_{n+1}=\min \left(\gamma, k^{5}\right)$ if $2 \leqslant n+1 \leqslant 4$, and

(b) $V_{0}^{n+1}=\Gamma_{n+1}^{(5)}(V)$, given by (4.8), as an initial guess for $V^{n+1}$ and a corresponding tolerance $\beta_{n+1}=\min (\gamma, k)$ if $5 \leqslant n+1 \leqslant J$, and, if we assume that $\left(q_{1}, q_{2}\right)$ $\in \overline{\mathscr{R}}$ so that (2.23), (2.24) hold, then for compatible $u^{0} \in H^{\mu}, u_{t}^{0} \in H^{\mu-1}, \mu=$ $\max (7, r+2)$, there exists a constant $C$, independent of $h, k, u$, such that for $0 \leqslant n \leqslant J$,

$$
\begin{aligned}
\left\|Z^{n}\right\| \leqslant & \left\|Z^{0}\right\|+C k^{-1}\left|\zeta^{0.1}\right|^{1 / 2} \\
& +C\left\{k^{4}\left(\left\|u^{0}\right\|_{7}+\left\|u_{t}^{0}\right\|_{6}\right)+h^{r}\left(\left\|u^{0}\right\|_{r+2}+\left\|u_{t}^{0}\right\|_{r+1}\right)\right\},
\end{aligned}
$$

where

$$
\zeta^{0.1} \text { is defined as } e^{0.1} \text { in (2.26) with } Z^{i} \text { instead of } E^{i}, \quad i=0,1 \text {. }
$$

Proof. The proof is largely identical to that of Theorem 2.1. The error equation is now, in view of (2.1) and (4.6),

$$
\mathscr{S}^{n}\left(Z^{n}\right)=-\mathscr{S}^{n}\left(W^{n}\right)+Q^{n+1}\left(V^{n+1}-\bar{V}^{n+1}\right), \quad 1 \leqslant n \leqslant J-1 .
$$

Taking the $L^{2}$-inner product of (4.11) with $Z^{n+1}-Z^{n-1}$, we see that (2.27) holds with $Z^{n}$ instead of $E^{n}$ and the added term $\left(Q^{n+1}\left(V^{n+1}-\bar{V}^{n+1}\right), Z^{n+1}-Z^{n-1}\right)$ in its right-hand side. The latter term may be estimated as follows. First note that (4.7), (4.4) and the triangle inequality imply that there is a $\gamma>0$ such that for $\beta_{n+1} \leqslant \gamma$

$$
\left\|\left(Q^{n+1}\right)^{1 / 2}\left(V^{n+1}-\bar{V}^{n+1}\right)\right\| \leqslant C \beta_{n+1}\left\|\left(Q^{n+1}\right)^{1 / 2}\left(V^{n+1}-\Gamma_{n+1}^{(i)}(V)\right)\right\|,
$$

where (and in sequel) $i=1$ or 5 depending on $n$ as in (4.8). It follows that for $1 \leqslant n \leqslant J-1$,

$$
\begin{aligned}
& \left|\left(Q^{n+1}\left(V^{n+1}-\bar{V}^{n+1}\right), Z^{n+1}-Z^{n-1}\right)\right| \\
& \leqslant\left\|\left(Q^{n+1}\right)^{1 / 2}\left(V^{n+1}-\bar{V}^{n+1}\right)\right\|\left\|\left(Q^{n+1}\right)^{1 / 2}\left(Z^{n+1}-Z^{n-1}\right)\right\| \\
& \leqslant C \beta_{n+1}\left\|\left(Q^{n+1}\right)^{1 / 2}\left(Z^{n+1}-Z^{n-1}\right)\right\| \\
& \quad \times\left\{\left\|\left(Q^{n+1}\right)^{1 / 2}\left(Z^{n+1}-\Gamma_{n+1}^{(i)}(Z)\right)\right\|\right. \\
& \left.+\left\|\left(Q^{n+1}\right)^{1 / 2}\left(W^{n+1}-\Gamma_{n+1}^{(i)}(W)\right)\right\|\right\} .
\end{aligned}
$$


Now, by $(4,8),(1.5),(1.10)$ it follows that for $1 \leqslant n \leqslant J-1$,

$$
\left\|\left(Q^{n+1}\right)^{1 / 2}\left(Z^{n+1}-\Gamma_{n+1}^{(i)}(Z)\right)\right\| \leqslant C \sum_{j=n-i+2}^{n+1}\left\|\left(Q^{j}\right)^{1 / 2}\left(Z^{j}-Z^{j-1}\right)\right\| .
$$

On the other hand, it is not hard to see, using the Cauchy-Schwarz inequality, (2.3),

(1.2) and (2.4) that for $1 \leqslant n \leqslant J-1$,

$$
\begin{aligned}
& \left\|\left(Q^{n+1}\right)^{1 / 2}\left(W^{n+1}-\Gamma_{n+1}^{(i)}(W)\right)\right\| \\
& \quad \leqslant C\left\|W^{n+1}-\Gamma_{n+1}^{(i)}(W)\right\|+C k^{2}\left\|L_{h}^{n+1}\left(W^{n+1}-\Gamma_{n+1}^{(i)}(W)\right)\right\| \\
& \quad \leqslant C k^{i}\left(\left\|u^{0}\right\|_{7}+\left\|u_{t}^{0}\right\|_{6}\right) .
\end{aligned}
$$

Hence, (4.13), (4.14), (4.15) and our hypotheses on $\beta_{n+1}$ imply, for $1 \leqslant n \leqslant J-1$,

$$
\begin{aligned}
& \left|\left(Q^{n+1}\left(V^{n+1}-\bar{V}^{n+1}\right), Z^{n+1}-Z^{n-1}\right)\right| \\
& \quad \leqslant C k \sum_{j=n-i+2}^{n+1}\left\|\left(Q^{j}\right)^{1 / 2}\left(Z^{j}-Z^{j-1}\right)\right\|^{2}+C k^{11}\left(\left\|u^{0}\right\|_{7}+\left\|u_{t}^{0}\right\|_{6}\right)^{2} .
\end{aligned}
$$

Continuing now the proof as in Theorem 2.1 and summing both sides of the analog of (2.27) with respect to $n$ from $n=1$ to $l, 1 \leqslant l \leqslant J-1$, we see, by (4.16), that the new term in the right-hand side of the sum, i.e.,

$$
\sum_{n=1}^{l}\left(Q^{n+1}\left(V^{n+1}-\bar{V}^{n+1}\right), Z^{n+1}-Z^{n-1}\right),
$$

may be bounded above by terms of the type that already appear in the right-hand side of (2.37) (with $E^{n}$ replaced by $Z^{n}$ and $e^{0.1}$ by $\zeta^{0,1}$ ), with the addition of the increased regularity term $C k^{2}\left[k^{4}\left(\left\|u^{0}\right\|_{7}+\left\|u_{t}^{0}\right\|_{6}\right)\right]^{2}$. The rest of the proof follows, mutatis mutandis, that of Theorem 2.1 and the result (4.9) follows.

Preconditioned iterative methods of the type described above may also be used to approximate the solution of the linear system represented by (3.6) in such a way so that the initial error term $\left\|Z^{0}\right\|+C k^{-1}\left|\zeta^{0,1}\right|^{1 / 2}$ in (4.9) is of optimal order $O\left(k^{4}+h^{r}\right)$. Following [6] we identify for the purposes of this exercise $\left\{H,(\cdot, \cdot)_{H}\right\}$ with $S_{h}^{2}=S_{h} \times S_{h}$, endowed with the $L^{2} \times L^{2}$-inner product which is denoted by $((\cdot, \cdot))$ and defined for $\Phi=\left[\phi_{1}, \phi_{2}\right]^{T}, \Psi=\left[\psi_{1}, \psi_{2}\right]^{T} \in S_{h}^{2}$ as

$$
((\Phi, \Psi))=\left(\phi_{1}, \psi_{1}\right)+\left(\phi_{2}, \psi_{2}\right) \text {. }
$$

We put $\||\cdot| \mid=((\cdot, \cdot))^{1 / 2}$. As our system $A x=b$ we now regard a form of weighted normal equations for (3.6), namely the system

$$
\left(\tilde{\mathscr{B}}^{1}\right)^{*} \mathscr{T}_{h}^{0} \tilde{\mathscr{B}}^{1} \mathscr{U}^{1}=\left(\tilde{\mathscr{B}}^{1}\right)^{*} \mathscr{T}_{h}^{0} \tilde{\mathscr{A}}^{0} \mathscr{W}^{0},
$$

where $\left(\tilde{\mathscr{B}}^{1}\right)^{*}$ is the adjoint of $\tilde{\mathscr{B}}^{1}$ on $S_{h}^{2}$ with respect to the $L^{2} \times L^{2}$-inner product $((\cdot, \cdot))$ and where $\mathscr{T}_{h}^{0}$ (is not the inverse of $\mathscr{L}_{h}^{0}$ but) is defined as

$$
\mathscr{T}_{h}^{0}=\left(\begin{array}{cc}
I & 0 \\
0 & T_{h}^{0}
\end{array}\right)
$$

Clearly, with this identification, $A$ is selfadjoint and positive-definite on $H$. As preconditioner we shall now consider a selfadjoint, positive-definite operator $A_{0}$ $={ }^{p} \mathscr{B}$, defined on $S_{h}^{2}$, such that $\left({ }^{p} \mathscr{B}\right)^{-1} \Phi$ is easy to find for $\Phi \in S_{h}^{2}$ and satisfying, for some constants $C, C^{\prime}$ and for $\Phi \in S_{h}^{2}$,

$$
C\left(\left({ }^{p} \mathscr{B} \Phi, \Phi\right)\right) \leqslant\left(\left(\left(\tilde{\mathscr{B}}^{1}\right)^{*} \mathscr{T}_{h}^{0} \mathscr{B}^{1} \Phi, \Phi\right)\right) \leqslant C^{\prime}\left(\left({ }^{p} \mathscr{B} \Phi, \Phi\right)\right) .
$$


An example of such a ${ }^{p \mathscr{B}}$ is given by the following result whose proof follows from Lemma 5.1 of [6].

LEMMA 4.2. For $\beta>0$ the operator

$$
p_{\mathscr{B}}=\left(\begin{array}{cc}
\left(I+\beta k^{2} L_{h}^{0}\right)^{2} & 0 \\
0 & \left(I+\beta k^{2} L_{h}^{0}\right) T_{h}^{1}\left(I+\beta k^{2} L_{h}^{0}\right)
\end{array}\right)
$$

defines a suitable preconditioner for the iterative solution of the linear system (4.18), that satisfies (4.20).

(It is evident that we intend to take the same $\beta$ in (4.21) and (4.5).)

For consistency in notation we now let $\overline{\mathscr{V}}^{1}\left(=\mathscr{U}^{1}\right)$ be the exact solution of (4.18) and $\mathscr{V}^{1}$ be its approximation, obtained by applying $p_{1}$ steps of an iterative method that satisfies (i), (ii) in our present context, with a preconditioner ${ }^{p} \mathscr{B}$ that satisfies (4.20), so that, for a preassigned tolerance $\beta_{1}$, we have

$$
\left\|\left({ }^{p} \mathscr{B}\right)^{1 / 2}\left(\overline{\mathscr{V}}^{1}-\mathscr{V}^{1}\right)\right\| \leqslant \beta_{1}\left\|\left({ }^{p} \mathscr{B}\right)^{1 / 2}\left(\overline{\mathscr{V}}^{1}-\mathscr{V}_{0}^{1}\right)\right\|,
$$

where $\mathscr{V}_{0}^{1}$ is the initial guess for the iteration. The next result guarantees optimal rate of convergence for such an iteration in $p_{1}=O\left(\ln \left(k^{-1}\right)\right)$ steps, in general.

Proposition 4.2. There exists $\gamma>0$ such that if $\mathscr{V}^{1}=\left[V_{1}^{1}, V_{2}^{1}\right]^{T}$ is the approximation to the solution $\overline{\mathscr{V}}^{1}\left(=\mathscr{U}^{1}\right)$ of $(4.18)$, obtained by a preconditioned iterative method that satisfies properties (i), (ii) above when $\left\{H,(\cdot, \cdot)_{H}\right\}=\left\{S_{h}^{2},((\cdot, \cdot))\right\}$ using a preconditioner ${ }^{P \mathscr{B}}$ satisfying (4.20), the initial guess $\mathscr{V}_{0}^{1}=\mathscr{W}^{0}$ and a tolerance $\beta_{1}=$ $\min \left(\gamma, k^{4}\right)$ then, if we take $V^{0}=W^{0}$ and $V^{1}=V_{1}^{1}$, we have, for compatible $u^{0} \in H^{\mu}$, $u_{t}^{0} \in H^{\mu-1}, \mu=\max (6, r+2)$, that there exists a constant $C$, independent of $h, k$ and $u$, such that

$$
\left\|Z^{0}\right\|+k^{-1}\left|\zeta^{0.1}\right|^{1 / 2} \leqslant C\left\{k^{4}\left(\left\|u^{0}\right\|_{6}+\left\|u_{t}^{0}\right\|_{5}\right)+h^{r}\left(\left\|u^{0}\right\|_{r+2}+\left\|u_{t}^{0}\right\|_{r+1}\right)\right\},
$$

where $\zeta^{0,1}$ has been defined by (4.10).

Proof. We shall just sketch the proof briefly. Define $\mathscr{Z}^{1}=\mathscr{V}^{1}-\mathscr{W}^{1}$. It follows from (3.6) (with $\mathscr{U}^{1}=\overline{\mathscr{V}}^{1}$ ) that

$$
\begin{aligned}
\left\|\mathscr{B}^{1} \mathscr{Z}^{1}\right\|_{1} \leqslant & \left\|\left(\mathscr{B}^{1}-\tilde{\mathscr{B}}^{1}\right) \mathscr{Z}^{1}\right\|_{1}+\left\|\tilde{\mathscr{B}}^{1} \mathscr{W}^{1}-\tilde{\mathscr{A}}^{0} \mathscr{W}^{0}\right\|_{1} \\
& +\left\|\tilde{\mathscr{B}}^{1}\left(\overline{\mathscr{V}}^{1}-\mathscr{V}^{1}\right)\right\|_{1} .
\end{aligned}
$$

Now, as, e.g., in Lemma 4.3 of [6], we obtain

$$
\left\|\left(\mathscr{B}^{1}-\tilde{\mathscr{B}}^{1}\right) \mathscr{Z}^{1}\right\|_{1} \leqslant C k\left\|\mathscr{B}^{1} \mathscr{Z}^{1}\right\|_{1} .
$$

Also, by Lemma 4.4 of [6] we have the consistency result

$$
\left\|\tilde{\mathscr{B}}^{1} \mathscr{W}^{1}-\tilde{\mathscr{A}}^{0} \mathscr{W}^{0}\right\|_{1} \leqslant C k \sigma_{k, h},
$$

where $\sigma_{k, h}$ has been defined by (2.35). We may also conclude, by methods similar to those used in Theorem 5.1 of [6], that there exists $\gamma>0$ such that, for $\beta_{1}=$ $\min \left(\gamma, k^{4}\right)$,

$$
\left\|\tilde{\mathscr{B}}^{1}\left(\overline{\mathscr{V}}^{1}-\mathscr{V}^{1}\right)\right\|_{1} \leqslant C k\left\|\mathscr{B}^{1} \mathscr{Z}^{1}\right\|_{1}+C k^{5}\left(\left\|u^{0}\right\|_{4}+\left\|u_{t}^{0}\right\|_{3}\right) .
$$

Finally, (4.24)-(4.27) imply (since $Z^{1}=Z_{1}^{1}, Z^{0}=0$ ), as in the proof of Proposition 3.1 , that (3.1) holds with $Z^{1}$ instead of $E^{1}$. (4.23) then follows. 
The results of Propositions 4.1 and 4.2 may now be combined to give a convergence theorem for the variant of the base scheme that uses $V^{0}=W^{0}$ and preconditioned iterative methods to compute the approximations $V^{n}$ to $u^{n}$, for $1 \leqslant n \leqslant J$.

THEOREM 4.1. There exists $\gamma>0$ such that if $\left\{V^{n}\right\}_{n=0}^{J}$ are computed by the algorithms described in Propositions 4.1 and 4.2 and the combined hypotheses of these propositions hold, then there exists a constant $C$, independent of $h, k$, and $u$, such that for $0 \leqslant n \leqslant J$,

$$
\left\|V^{n}-u^{n}\right\| \leqslant C\left\{k^{4}\left(\left\|u^{0}\right\|_{7}+\left\|u_{t}^{0}\right\|_{6}\right)+h^{r}\left(\left\|u^{0}\right\|_{r+2}+\left\|u_{t}^{0}\right\|_{r+1}\right)\right\} .
$$

5. Replacing Derivatives of $L_{h}$ by Difference Quotients. It is possible to replace the derivatives $L_{h}^{(2) n}, L_{h}^{(1) n}$ of the operator $L_{h}$ that occur in the base scheme (2.1), if $q_{1} \neq 1 / 12$, by difference quotients involving values of $L_{h}^{n}$ for $0 \leqslant n \leqslant J$. As a result the computational cost will decrease because, as (2.1) stands now, these derivatives have to be evaluated at each step for the computation of the right-hand side of the linear system implied by (2.1). This task may be easily accomplished in our case (of a linear, time-dependent coefficient problem) by replacing $L_{h}^{(1) n}$ by, e.g.,

$$
\delta L_{h}^{n} \equiv\left(L_{h}^{n+1}-L_{h}^{n-1}\right) / 2 k, \quad 1 \leqslant n \leqslant J-1,
$$

and $L_{h}^{(2) n}$ by

$$
\delta^{2} L_{h}^{n} \equiv\left(L_{h}^{n+1}-2 L_{h}^{n}+L_{h}^{n-1}\right) / k^{2}, \quad 1 \leqslant n \leqslant J-1 .
$$

Then, the scheme (2.1) is replaced by the following: given $U_{\delta}^{0}, U_{\delta}^{1}$ in $S_{h}$, we seek $\left\{U_{\delta}^{n}\right\}_{n=2}^{J}$ in $S_{h}$ satisfying, for $1 \leqslant n \leqslant J-1$,

$$
\begin{aligned}
\mathscr{S}_{\delta}^{n}\left(U_{\delta}^{n}\right) \equiv & Q^{n+1} U_{\delta}^{n+1}+Q^{n-1} U_{\delta}^{n-1}-2 P^{n} U_{\delta}^{n}-k^{4}\left(q_{1}-\frac{1}{12}\right) \\
& \times\left\{\left(\delta^{2} L_{h}^{n}\right) U_{\delta}^{n}+2\left(\delta L_{h}^{n}\right)\left[\frac{1}{k}\left(U_{\delta}^{n}-U_{\delta}^{n-1}\right)-\frac{k}{2} L_{h}^{n} U_{\delta}^{n}\right]\right\}=0 .
\end{aligned}
$$

We indicate without proof the steps in the convergence analysis of the scheme (5.3).

We now state the "difference" analog of Theorem 2.1. In sequel we put $E_{\delta}^{n}=$ $U_{\delta}^{n}-W^{n}, 0 \leqslant n \leqslant J$.

Proposition 5.1. Let $U_{\delta}^{0}, U_{\delta}^{1}$ be given in $S_{h}$ and $\left\{U_{\delta}^{n}\right\}_{n=2}^{J}$ be the solution of (5.3). Then, under the remaining hypotheses of Theorem 2.1, it follows that the result of Theorem 2.1 holds if we replace $E^{n}$ by $E_{\delta}^{n}$ in (2.25) and (2.26).

To supply the initial value $U^{1}$ for (5.3) we can use a scheme similar to (3.6). We shall leave $\tilde{\mathscr{A}}^{0} \mathscr{W}^{0}$ as is-this necessitates the computation of $L_{h}^{(1)}(t)$ at $t=0$ only-but replace the operator $\tilde{\mathscr{B}}^{1}$ by $\tilde{\mathscr{B}}_{\delta}^{1}$, defined in terms of $\tilde{\mathscr{B}}^{1}, \mathscr{B}^{1}$ as

$$
\tilde{\mathscr{B}}_{\delta}^{1}=\mathscr{B}^{1}+\frac{k^{2}}{12} \Delta \mathscr{L}_{h}^{1}=\tilde{\mathscr{B}}^{1}+\frac{k^{2}}{12}\left(\Delta \mathscr{L}_{h}^{1}-\mathscr{L}_{h}^{(1) 1}\right),
$$

where, as it turns out, it is appropriate to take $\Delta \mathscr{L}_{h}^{1}$ as a third-order accurate difference approximation to $\mathscr{L}_{h}^{(1) 1}$. To fix ideas let

$$
\Delta \mathscr{L}_{h}^{1} \equiv\left(-2 \mathscr{L}_{h}^{0}-3 \mathscr{L}_{h}^{1}+6 \mathscr{L}_{h}^{2}-\mathscr{L}_{h}^{3}\right) / 6 k
$$

It may be seen by the proofs of Lemmata 4.2 and 4.3 of [6] that for $\Phi \in S_{h}^{2}$,

$$
\left\|\left(\tilde{\mathscr{B}}_{\delta}^{1}-\mathscr{B}^{1}\right) \Phi\right\|_{1} \leqslant C k^{2} \sup _{0 \leqslant \tau \leqslant 3 k}\left\|\mathscr{L}_{h}^{(1)}(\tau) \Phi\right\|_{1} \leqslant C k\left\|\mathscr{B}^{1} \Phi\right\|_{1},
$$


from which the invertibility of $\tilde{\mathscr{B}}_{\delta}^{1}$ follows for $k$ sufficiently small. We define now $\mathscr{U}_{\delta}^{1} \in S_{h}^{2}$ as the solution of

$$
\tilde{\mathscr{B}}_{\delta}^{1} \mathscr{U}_{\delta}^{1}=\tilde{\mathscr{A}}^{0} \mathscr{W}^{0} .
$$

Proposition 5.2. Let $U_{\delta}^{0}=W^{0}$ and $\mathscr{U}_{\delta}^{1}=\left[U_{\delta, 1}^{1}, U_{\delta, 2}^{1}\right]^{T}$ be the solution of (5.7). Define $U_{\delta}^{1}=U_{\delta, 1}^{1}$. Then, under the remaining hypotheses of Proposition 3.1, the result of Proposition 3.1 holds with $E^{i}$ replaced by $E_{\delta}^{i}, i=0,1$, in (3.8) and (2.26).

Combining now the results of Propositions 5.1 and 5.2, we have, in view of (2.3), the following optimal-order convergence result for the new base scheme.

THEOREM 5.1. Let $U_{\delta}^{0}, U_{\delta}^{1}$ be selected as in Proposition 5.2 and let $\left\{U_{\delta}^{n}\right\}_{n=2}^{J}$ be the solution of the scheme (5.3). Then, under the combined hypotheses of Propositions 5.1 and 5.2 we conclude that there exists a constant $C$, independent of $h, k$, and $u$, such that for $0 \leqslant n \leqslant J$,

$$
\left\|U^{n}-u^{n}\right\| \leqslant C\left\{k^{4}\left(\left\|u^{0}\right\|_{6}+\left\|u_{t}^{0}\right\|_{5}\right)+h^{r}\left(\left\|u^{0}\right\|_{r+2}+\left\|u_{t}^{0}\right\|_{r+1}\right)\right\} .
$$

The operators in the left-hand sides of the system (5.3) and (5.7) still change at each time step. In analogy to what was done in Section 4 we may again use preconditioned iterative methods for the efficient solution of these systems. Therefore, we describe below our final method, which combines difference quotients and preconditioned iterative procedures.

Consider first the difference analog of the linear system (4.18), i.e., the equation (we put $\overline{\mathscr{V}}_{\delta}^{1}=\mathscr{U}_{\delta}^{1}$ )

$$
\left(\tilde{\mathscr{B}}_{\delta}^{1}\right)^{*} \mathscr{T}_{h}^{0} \tilde{\mathscr{B}}_{\delta}^{1} \overline{\mathscr{V}}_{\delta}^{1}=\left(\tilde{\mathscr{B}}_{\delta}^{1}\right)^{*} \mathscr{T}_{h}^{0} \tilde{\mathscr{A}}^{0} \mathscr{W}^{0},
$$

where $\left(\tilde{\mathscr{B}}_{\delta}^{1}\right)^{*}$ is the adjoint of $\tilde{\mathscr{B}}_{\delta}^{1}$ in $S_{h}^{2}$ with respect to the inner product $((\cdot, \cdot))$. To apply to (5.9) a preconditioned iterative method, such as the ones described in Section 4 , we identify $\left\{H,(\cdot, \cdot)_{H}\right\}$ with $\left\{S_{h}^{2},((\cdot, \cdot))\right\}$ and regard (5.9) as the system $A x=b$. It can be shown (we omit the proof) that an example of a suitable preconditioner that satisfies (4.1) in our context is furnished again by the operator

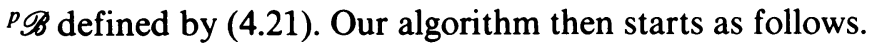

Step 1. Construct an approximation $\mathscr{V}_{\delta}^{1}=\left[V_{\delta, 1}^{1}, V_{\delta, 2}^{1}\right]^{T}$ in $S_{h}^{2}$ to the exact solution $\overline{\mathscr{V}}_{\delta}^{1}$ of (5.9) by using a preconditioned iterative method that satisfies properties (i) and (ii) of Section 4 - where $\left\{H,(\cdot, \cdot)_{H}\right\}=\left\{S_{h}^{2},((\cdot, \cdot))\right\}-$, with preconditioner ${ }^{p} \mathscr{B}$ defined by (4.21), using $\mathscr{W}^{0}$ as initial guess for the iteration and a tolerance $\beta_{1}=\min \left(\gamma, k^{4}\right)$ with $\gamma$ sufficiently small. (This will require in general $O\left(\ln \left(k^{-1}\right)\right.$ ) iterations.) Put $V_{\delta}^{0}=W^{0}, V_{\delta}^{1}=\mathscr{V}_{\delta, 1}^{1}$.

Now, to compute $\left\{V_{\delta}^{n}\right\}_{n=2}^{J}$, consider the "difference" analog of the time-stepping scheme (4.6), i.e., let $\bar{V}_{\delta}^{n}, 1 \leqslant n \leqslant J-1$, be the (exact) solution of

$$
\begin{aligned}
Q^{n+1} \bar{V}_{\delta}^{n+1}= & 2 P^{n} V_{\delta}^{n}-Q^{n-1} V_{\delta}^{n-1}+k^{4}\left(q_{1}-\frac{1}{12}\right) \\
& \times\left\{\left(\delta^{2} L_{h}^{n}\right) V_{\delta}^{n}+2\left(\delta L_{h}^{n}\right)\left[\frac{1}{k}\left(V_{\delta}^{n}-V_{\delta}^{n-1}\right)-\frac{k}{2} L_{h}^{n} V_{\delta}^{n}\right]\right\},
\end{aligned}
$$

which we regard as the system $A x=b$ in the notation of Section 4 identifying now $\{H,(\cdot, \cdot)\}$ with $\left\{S_{h},(\cdot, \cdot)\right\}$. An example of a suitable preconditioner that satisfies 
(4.4) is of course again ${ }^{p} Q$ given by (4.5). The rest of the algorithm is then:

Step 2. With $V_{\delta}^{0}, V_{\delta}^{1}$ constructed in Step 1 , compute for each $n, 1 \leqslant n \leqslant J-1$, an approximation $V^{n+1}$ to the exact solution $\bar{V}^{n+1}$ of (5.10) using a preconditioned iterative method that satisfies properties (i) and (ii) of Section 4-where $\left\{H,(\cdot, \cdot)_{H}\right\}=\left\{S_{h},(\cdot, \cdot)\right\}-$, with preconditioner ${ }^{p} Q$ defined by $(4.5)$, using $V_{0}^{n+1}$ $=\Gamma_{n+1}^{(i)}(V)$, given by (4.8), as initial guess for the iteration and tolerances

$$
\beta_{n+1}= \begin{cases}\min \left(\gamma, k^{5}\right) & \text { if } 2 \leqslant n+1 \leqslant 4, \\ \min (\gamma, k) & \text { if } 5 \leqslant n+1 \leqslant J,\end{cases}
$$

with $\gamma$ sufficiently small. (This will require, in general, $O\left(\ln \left(k^{-1}\right)\right)$ iterations for each n.)

By methods similar to those used in the proof of Theorem 4.1 we can finally prove

THEOREM 5.2. There is a $\gamma>0$ such that if $\left\{V_{\delta}^{n}\right\}_{n=0}^{J}$ are computed by the algorithm described in Steps 1 and 2 above and if the remaining hypotheses of Theorem 4.1 hold, then there exists a constant $C$, independent of $h, k$, and $u$, such that for $0 \leqslant n \leqslant J$,

$$
\left\|V_{\delta}^{n}-u^{n}\right\| \leqslant C\left\{k^{4}\left(\left\|u^{0}\right\|_{7}+\left\|u_{t}^{0}\right\|_{6}\right)+h^{r}\left(\left\|u^{0}\right\|_{r+2}+\left\|u_{t}^{0}\right\|_{r+1}\right)\right\}
$$

Department of Mathematics

University of Tennessee

Knoxville, Tennessee 37996-1300

Department of Mathematics

University of Crete

Iraklion, Crete, Greece

1. O. AXELSSON, "Solution of linear systems of equations: Iterative methods," Sparse Matrix Techniques (V. A. Barker, ed.), Lecture Notes in Math., vol. 572, Springer-Verlag, Berlin and New York, 1976, pp. 1-51.

2. G. A. BAKer \& J. H. Bramble, "Semidiscrete and single step fully discrete approximations for second order hyperbolic equations," RAIRO Anal. Numer., v. 13, 1979, pp. 75-100.

3. G. A. Baker, V. A. Dougalis \& S. M. Serbin, "An approximation theorem for second-order hyperbolic equations," Numer. Math., v. 35, 1980, pp. 127-142.

4. G. A. Baker, V. A. Dougalis \& S. M. Serbin, "High order accurate, two-step approximations for hyperbolic equations," RAIRO Anal. Numér., v. 13, 1979, pp. 201-226.

5. L. A. BALES, "Semidiscrete and single step fully discrete approximations for second order hyperbolic equations with time-dependent coefficients," Math. Comp., v. 43, 1984, pp. 383-414.

6. L. A. BALES, "Higher order single step fully discrete approximations for second order hyperbolic equations with time dependent coefficients," SIAM J. Numer. Anal. (To appear.)

7. J. H. Bramble \& P. H. Sammon, "Efficient higher order single step methods for parabolic problems: Part I," Math. Comp., v. 35, 1980, pp. 655-677.

8. J. H. Bramble, A. H. Schatz, V. Thomee \& L. B. Wahlbin, "Some convergence estimates for semidiscrete Galerkin type approximations for parabolic equations," SIAM J. Numer. Anal., v. 14, 1977, pp. $218-241$.

9. J. Douglas, JR., T. Dupont \& R. E. Ewing, “Incomplete iteration for time-stepping a Galerkin method for a quasilinear parabolic problem," SIAM J. Numer. Anal., v. 16, 1979, pp. 503-522.

10. R. E. EwING, "On efficient time-stepping methods for nonlinear partial differential equations," Comput. Math. Appl., v. 6, 1980, pp. 1-13.

11. P. H. SAmmon, "Convergence estimates for semidiscrete parabolic equation approximations," SIAM J. Numer. Anal., v. 19, 1982, pp. 68-92.

12. S. M. SERBIN, "Rational approximation of trigonometric matrices with applications to second-order systems of differential equations," Appl. Math. Comput., v. 5, 1979, pp. 59-92.

13. S. M. SERBin, "Some cosine schemes for second-order systems of O.D.E.'s with time-varying coefficients," SIAM J. Sci. Statist. Comput., v. 6, 1985, pp. 61-68. 